\title{
Trajectory-Based Loads for the Ares I-X Test Flight Vehicle
}

\author{
R.F. Vause ${ }^{1}$ and Brett R. Starr ${ }^{2}$ \\ NASA Langley Research Center, Hampton, VA, 23681
}

In trajectory-based loads, the structural engineer treats each point on the trajectory as a load case. Distributed aero, inertial, and propulsion forces are developed for the structural model which are equivalent to the integrated values of the trajectory model. Free-body diagrams are then used to solve for the internal forces, or loads, that keep the applied aero, inertial, and propulsion forces in dynamic equilibrium. There are several advantages to using trajectory-based loads. First, consistency is maintained between the integrated equilibrium equations of the trajectory analysis and the distributed equilibrium equations of the structural analysis. Second, the structural loads equations are tied to the uncertainty model for the trajectory systems analysis model. Atmosphere, aero, propulsion, mass property, and controls uncertainty models all feed into the dispersions that are generated for the trajectory systems analysis model. Changes in any of these input models will affect structural loads response. The trajectory systems model manages these inputs as well as the output from the structural model over thousands of dispersed cases. Large structural models with hundreds of thousands of degrees of freedom would execute too slowly to be an efficient part of several thousand system analyses. Trajectory-based loads provide a means for the structures discipline to be included in the integrated systems analysis. Successful applications of trajectorybased loads methods for the Ares I-X vehicle are covered in this paper. Preliminary design loads were based on 2000 trajectories using Monte Carlo dispersions. Range safety loads were tied to 8423 malfunction turn trajectories. In addition, active control system loads were based on 2000 preflight trajectories using Monte Carlo dispersions.

$\begin{array}{ll} & \\ M & =\text { vehicle bending moment } \\ N_{s} & =\text { maximum shear stress running load } \\ N_{x m a x} & =\text { maximum normal stress running load } \\ N_{x m i n} & =\text { minimum normal stress running load } \\ P & =\text { vehicle axial force } \\ \text { qalpha } & =\text { product of dynamic pressure and vehicle angle of attack } \\ r & =\text { vehicle radius } \\ T & =\text { vehicle torsion moment } \\ V & =\text { vehicle shear force }\end{array}$

\section{Introduction}

Planning for the Ares I-X (AIX) flight test vehicle (FTV) began in the late summer of 2006. The AIX FTV was to be similar to the Constellation Program's Ares I Crew Launch Vehicle in terms of aerodynamics and mass properties. The 320-foot FTV consisted of a first stage (FS), upper stage (US) mass simulator, and crew modulelaunch abort system (CM-LAS) mass simulator (Fig. 1). The FS was an existing shuttle four-segment reusable solid rocket motor (RSRM) with an inert fifth-segment extension to maintain similarity with the Ares I five-segment RSRM. The US and CM-LAS simulators, as well as the forward structures of FS, were all new hardware which had to be designed and fabricated prior to the planned launch date of Spring 2009. The objectives of the AIX program were to demonstrate controllability of the slender vehicle during flight and recovery of the five-segment FS after separation.

The FS, US, and CM-LAS design and fabrication were handled by integrated product teams (IPTs). The FS structure was the responsibility of Alliant Techsystems (ATK) and NASA Marshall Space Flight Center (MSFC),

\footnotetext{
${ }^{1}$ Structural Engineer, Vehicle Analysis Branch, Mail Stop 451, AIAA Member.

${ }^{2}$ Aerospace Engineer, Vehicle Analysis Branch, Mail Stop 451, AIAA Member.
} 
while the engineering for the US simulator was handled by NASA Glenn Research Center (GRC). NASA Langley Research Center (LaRC) developed the CM-LAS simulator. System Engineering and Integration (SEI) was responsible for analyses related to the FS, US, and CM-LAS functioning as an integrated system. The SEI Office was located at LaRC. System assembly and launch was at NASA Kennedy Space Center (KSC).

The SEI trajectory team developed a system level vehicle model to simulate the AIX flight performance. Each time point on a trajectory run represents a rigid body in dynamic equilibrium under integrated aero, inertial, and propulsion applied forces. There is a considerable investment of work to support a trajectory analysis. The mass properties group has to make sure the desired propellant, payload, and propulsion system can be packaged. Aero databases are generated to estimate lift and drag forces as a function of Mach number and angles of attack. Thrust inputs from the propulsion group are usually based on statistical data from ground test engine firings. Atmosphere information including winds aloft is pulled from the Global Reference Atmosphere Model (GRAM). All of this data with associated uncertainties is fed into the trajectory systems analysis model that estimates vehicle performance.

A structural analysis should be viewed as a distributed version of the equilibrium equations from the integrated trajectory analysis. The structural engineer is taking advantage of the considerable amount of work that has gone into setting up the trajectory analysis. Each trajectory time point represents a potential load case with distributed inertial, aero, and propulsion applied forces. The distributed applied forces of the structural model should always equal the trajectory applied forces when they are integrated about a defined reference point such as the vehicle center of gravity.

The distributed applied forces on the structural model are kept in equilibrium by the structure internal forces (axial force, shears, bending moments, torsion). Since these internal forces are usually associated with a vehicle cross section, they are often referred to as section loads. Design engineers refer to internal forces as design loads or simply loads. Vehicle design load forces and moments are combined to form running loads, which are forces per unit width of structure.

$$
\begin{aligned}
& N_{x \max }=P /(2 \pi r)+M /\left(\pi r^{2}\right) \\
& N_{x \min }=P /(2 \pi r)-M /\left(\pi r^{2}\right) \\
& N_{s}=T /\left(2 \pi r^{2}\right)+2 V /(\pi r)
\end{aligned}
$$

These strength of materials equations assume a circular cross section with a uniform distribution of axial force and torsion. Bending moments vary linearly over the cross section. Running loads can be converted to stresses by dividing by the equivalent cross section wall thickness. Additional details on design loads for aerospace structures can be found in Ref. 1.

All vehicle motion and associated loads consist of rigid-body and flex-body response. The combined rigid-body and flex-body load can be approximated as the rigid-body load increased by a dynamic load factor (DLF) to account for flex-body effects. Approximating the combined load with an adjusted rigid-body load simplifies the analysis since rigid-body analyses do not need stiffness distributions, mode shapes, or frequencies. To account for flex-body effects, a DLF of 1.5 was applied to the rigid-body structural load results. The basis for a DLF of 1.5 is illustrated in Fig. 2, which was taken from Ref. 2. A large percentage of loads that are applied to a launch vehicle can be categorized as quasi-static or single pulse loads. If the period of the applied force $\left(t_{d}\right)$ is large compared to the period of the structure response (T), then the applied force is considered static (DLF=1.0). For applied forces with a shorter period, the DLF can go as high as 2.0. The choice of DLF=1.5 for AIX design loads was based on the average of the static DLF and the peak response DLF.

It is important to recognize the difference between single pulse applied forces and frequency-driven applied forces which can tune to a structural response frequency (Fig. 3). Simple DLFs cannot account for the dramatic response amplification if the forcing function frequency is close to a structural frequency. Energy is continually added to the system by the forcing function with the only energy dissipation coming from the velocity-dependent damping forces. The curves in Fig. 3 represent the response at different frequency ratios for different fractions of critical damping. The response at a resonant frequency ratio of 1.0 is infinite if there is no damping present. The AIX vehicle structure was assumed to have a damping ratio of 0.01 , which would have a maximum response amplification of 50 at a resonant frequency ratio. Some applied forces have both quasi-static content, as well as a defined frequency content. The average motor dome forces of Fig. 4 are treated as quasi-static. There is also a lower amplitude oscillating thrust component that is driven by acoustic modes in the motor.

In trajectory-based loads, the structural engineer treats each point on the trajectory as a load case. Distributed aero, inertial, and propulsion forces are developed for the structural model which are equivalent to the integrated 
values of the trajectory model. Free-body diagrams are then used to solve for the internal forces, or loads, that keep the applied aero, inertial, and propulsion forces in dynamic equilibrium. There are several advantages to using trajectory-based loads. First, consistency is maintained between the integrated equilibrium equations of the trajectory analysis and the distributed equilibrium equations of the structural analysis. The structural loads calculations performed at each time point of the trajectory analysis are tied to the equilibrium equations that solve for vehicle position, velocity, and acceleration. Second, the structural loads equations are tied to the uncertainty model for the trajectory systems analysis model. Atmosphere, aero, propulsion, mass property, and controls uncertainty models all feed into the dispersions that are generated for the trajectory systems analysis model. Changes in any of these input models will affect structural loads response. The trajectory systems model manages these inputs as well as the output from the structural model. The structural model must execute quickly to accommodate thousands of trajectory analyses for Monte Carlo dispersions or postulated failure scenarios. Large structural models with hundreds of thousands of degrees of freedom would execute too slowly to be an efficient part of several thousand system analyses. Without trajectory-based loads, the structures discipline would not be a part of the integrated systems analysis model. Successful applications of trajectory-based loads methods for the Ares I-X vehicle are covered in this paper. Preliminary design loads were based on 2000 trajectories using Monte Carlo dispersions. Range safety loads were tied to 8423 malfunction turn trajectories. Active control system loads were based on 2000 preflight trajectories using Monte Carlo dispersions.

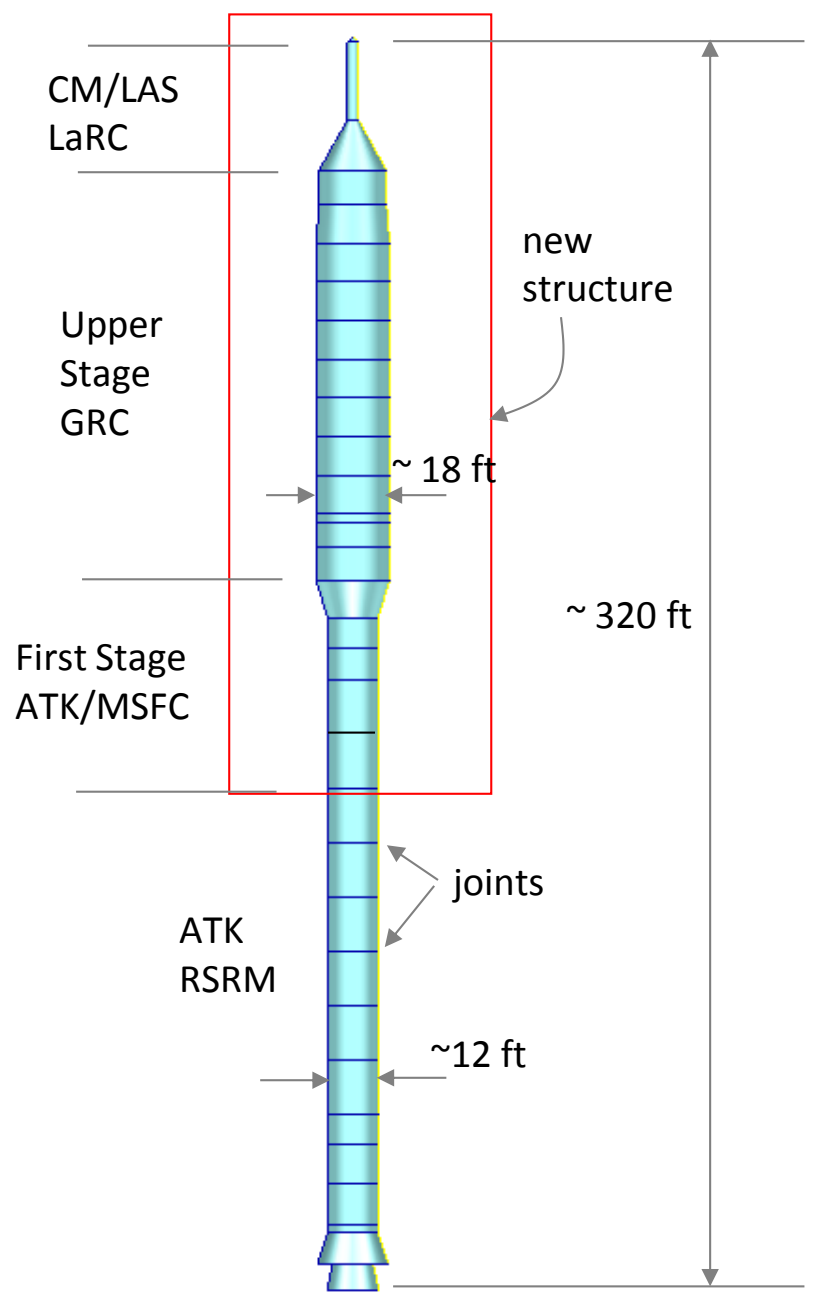

Figure 1. Ares I-X Flight Test Vehicle. 


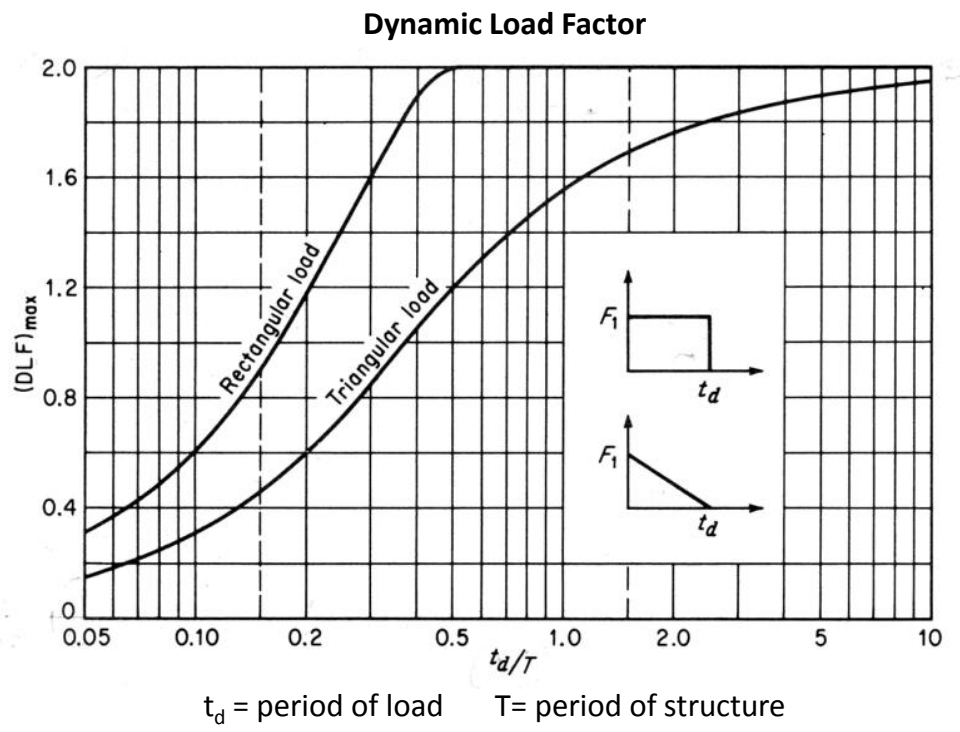

Figure 2. Dynamic Load Factor for Single-Pulse Loads.

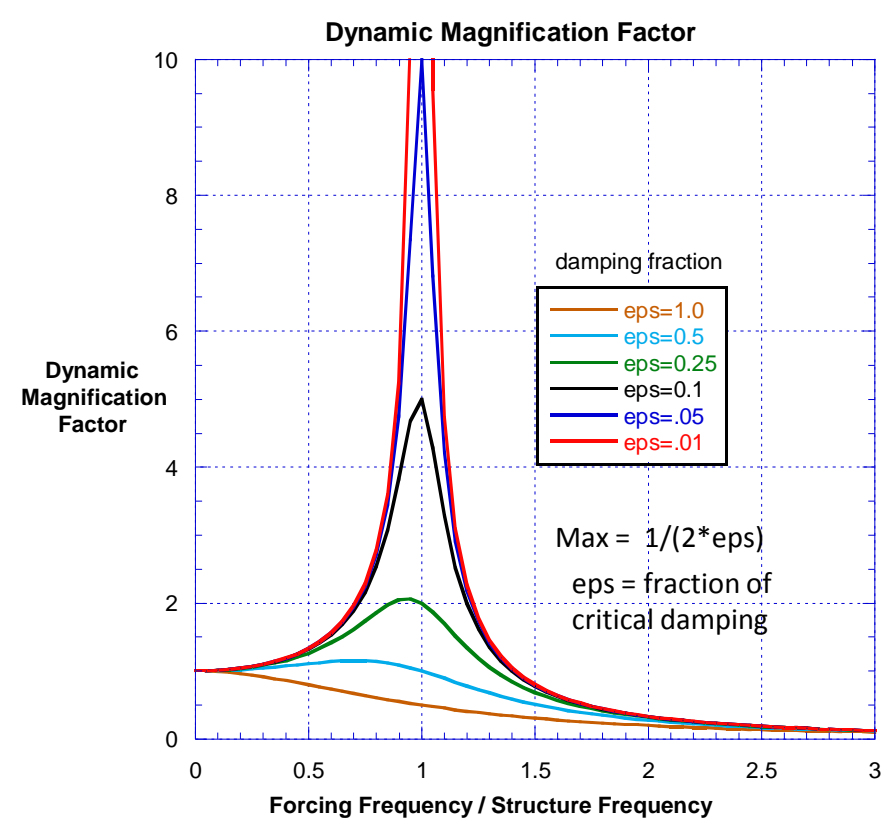

Figure 3. Tuning of Forcing Function Frequency and Structural Frequency 


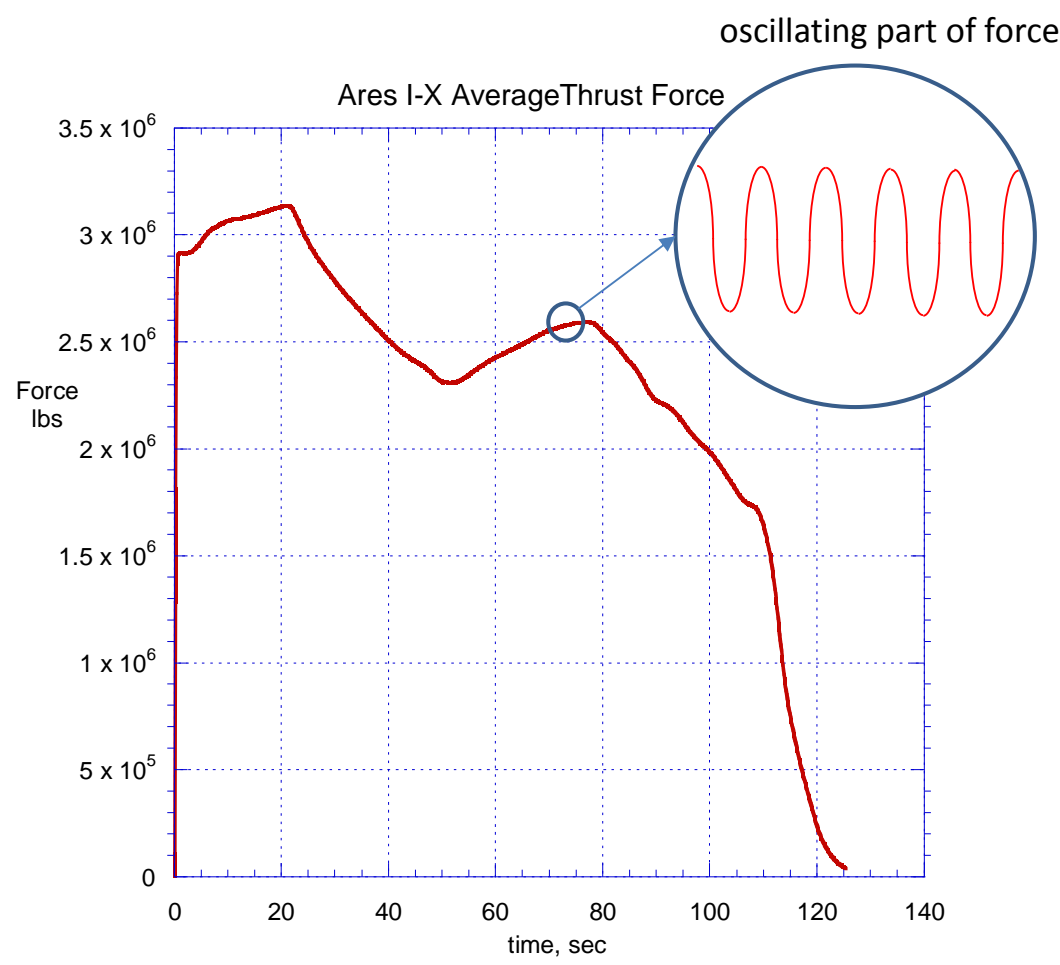

Figure 4. Average Thrust Force and Oscillating Thrust Force

\section{Preliminary Design Loads}

In early 2007, the AIX IPTs requested design loads from SEI so they could proceed with sizing and evaluating structure. As noted earlier, all the structure above the FS four-segment motor was new hardware which had to be analyzed, designed, fabricated, and assembled. Trajectory systems models for Monte Carlo analyses were already in place using integrated mass properties, integrated aero databases, and integrated thrust. All of these values varied with time and the current state of the vehicle (velocity, angle of attack, propellant burned). The SEI Trajectory and Structures Groups developed a trajectory-based loads analysis to define the requirements for FTV design loads. The preliminary structural loads models were integrated into the systems analysis trajectory simulation. The integrated analysis determined the structural loads at each station along the vehicle at each time point of every dispersed trajectory simulation. The envelope of loads resulting from the Monte Carlo analyses would bound the ascent loads used to define the design load requirements.

Integrating the loads process would entail developing time-dependent spatial distributions for mass, aero forces, and propulsion. The integrated applied forces and vehicle state information would be passed to the structures code at each time point of each trajectory. Overall vehicle equilibrium would then be checked before section cuts and free-body diagrams were used to solve for vehicle internal forces. The last step of the process was the application of a DLF to account for the effects of flex-body dynamics. All structural load calculations were done in Fortran to take advantage of existing subroutines.

There was considerable uncertainty in mass distribution at this point in the AIX project. The IPTs updated their mass property estimates for each manufacturing segment every few months. There was even more uncertainty in the assumed stiffness for the proposed hardware. Therefore, a simple structural model was created where the geometry, mass, and stiffness for each FS, US, and CM-LAS segment could be changed quickly. Details of the segment mass and stiffness model are shown in Fig. 5. Multiple models were created to accommodate geometry changes, ballast distribution studies, separation joint location, and material changes. Each segment was assumed to be axisymmetric 
with a possible linear variation of mass. The segment weight, diameter, and thickness information were combined with material properties to produce stiffness information for Nastran beam models.

FS motor segment weights were split into fixed casing weight and time-dependent propellant weight. Propellant burn rates were based on tables provided by ATK. Structural model mass property estimates were checked against trajectory values, the AIX Systems Requirements Document, and the Nastran beam models discussed above. Early segment mass distributions for different trajectory time points are shown in Fig. 6. Note the steady decrease in motor segment mass with burn time. The propellant mass is over 70 percent of the total mass and its burning results in the vehicle center of gravity moving up the launch stack by about 700 inches.

The integrated aero coefficients from the AIX wind-tunnel tests were passed to the structural loads code at each time point on the trajectory. Coefficients for aero axial force, normal force, and pitching moment were defined as a function of roll angle and total angle of attack. For a distributed model, the aero normal force is not independent of pitching moment. Integrating the normal force distribution about the gimbal point should equal the pitching moment. Aero force distributions from computational fluid dynamics (CFD) must be combined with integrated aero forces from wind tunnel tests to generate meaningful aero line load databases. An early line mapping of Ares I-X aero coefficients from CFD analyses is shown in Fig. 7. There are sharp changes in axial and normal line coefficients where there is compressed flow (CM, aft skirt) and expanded flow (frustum). The agreement between wind tunnel coefficients and integrated CFD distributions was not good enough in early 2007 to support structural loads models. Hence, a judgment was made to develop a normal force distribution that satisfied both the integrated normal force and integrated pitching moment from the wind tunnel tests.

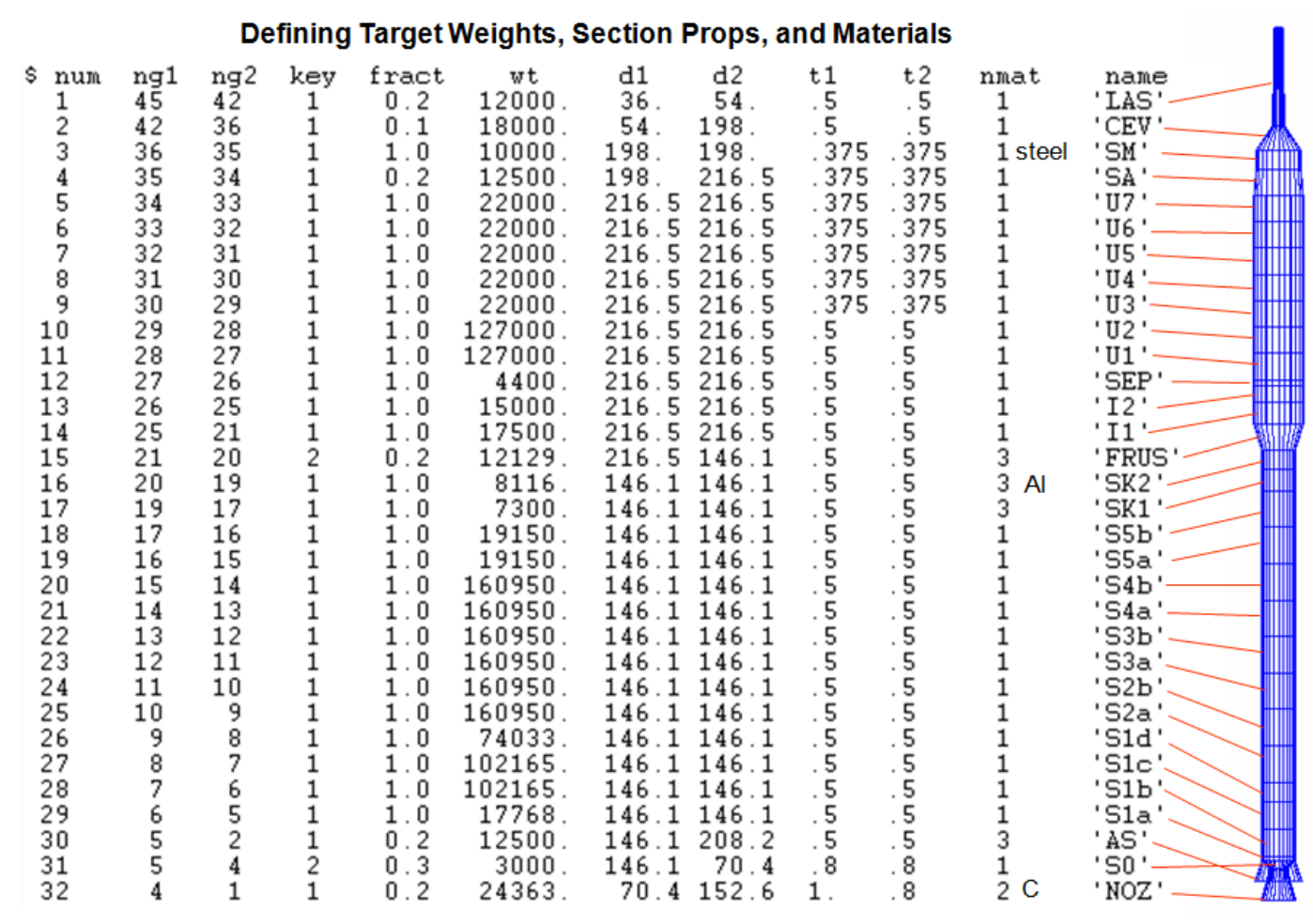

Figure 5. AIX Segment Mass and Stiffness Model from Spring 2007. 


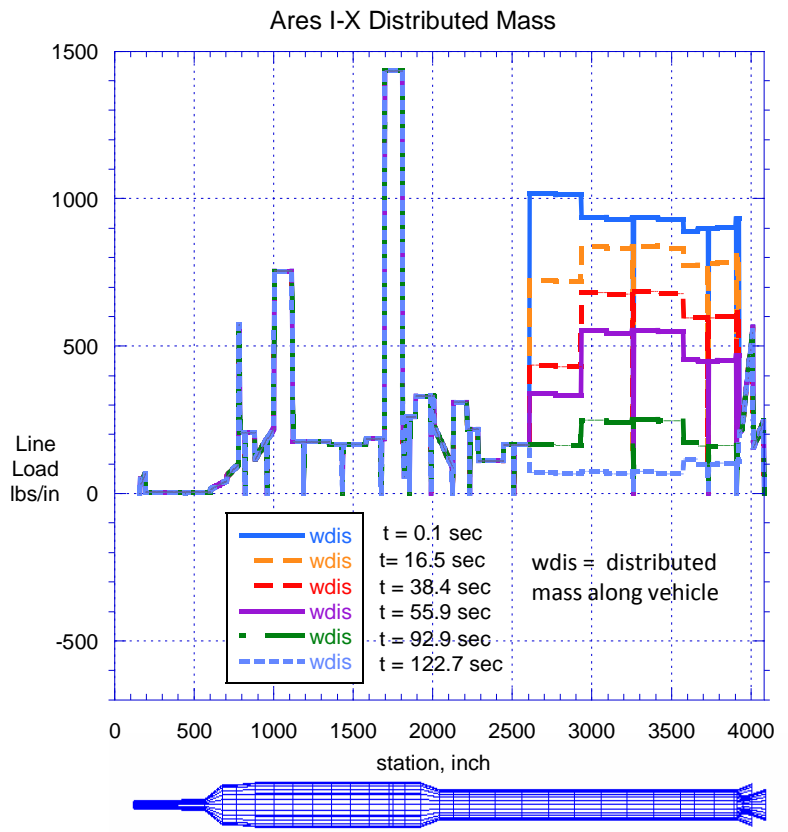

Figure 6. Distributed Mass as a Function of Burn Time.

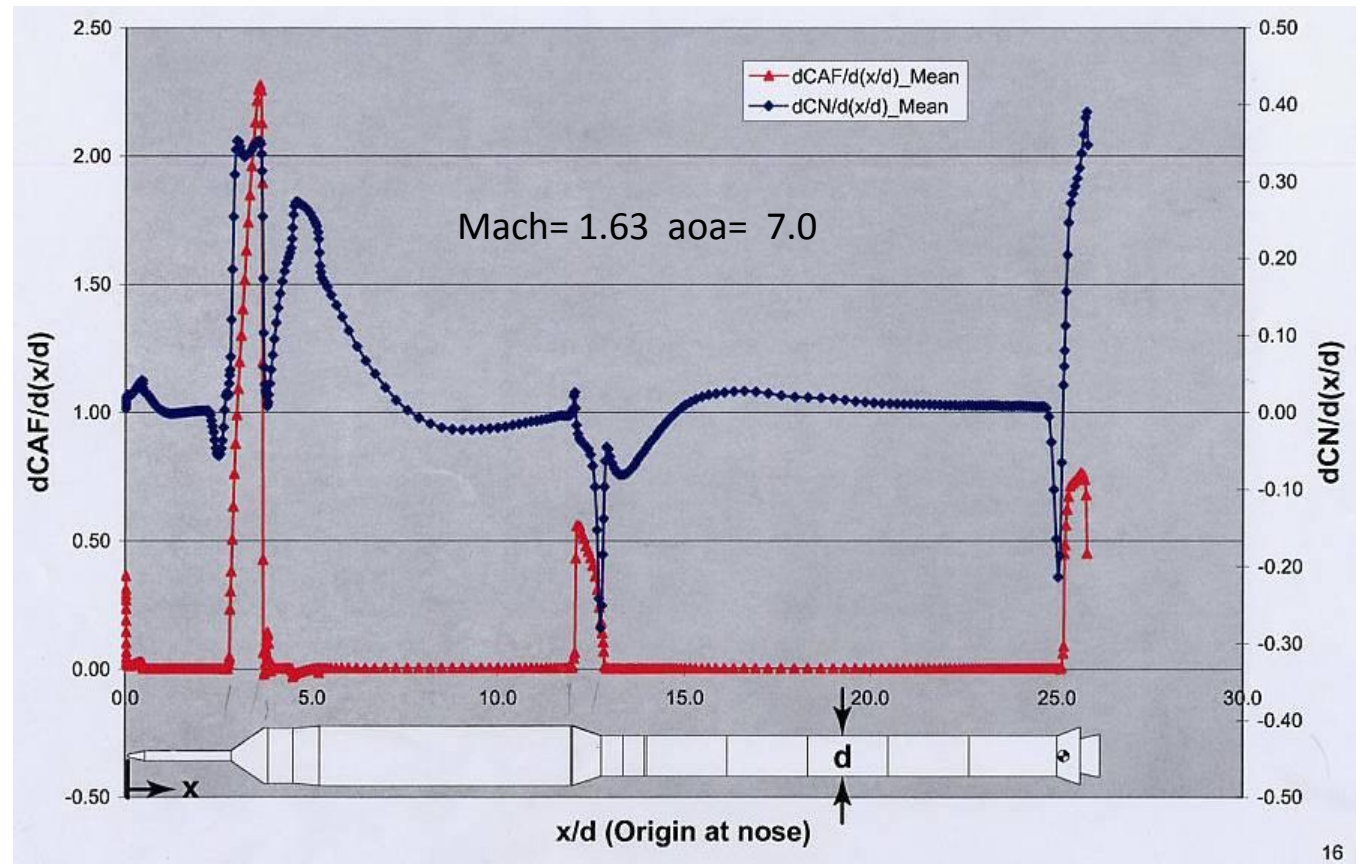

Figure 7. Aero Line Coefficients from CFD Analyses.

American Institute of Aeronautics and Astronautics 
The equivalent aero force systems are shown in Fig. 8. The normal force and pitching moment from the wind tunnel tests are shown referenced to the vehicle gimbal point. This force and moment can be replaced with an equivalent system at the vehicle center of gravity $(\mathrm{cg})$. This location is important because the gimbal trim force can be calculated directly using the cg aero moment and the arm between the gimbal and cg (angular acceleration assumed zero). A similar procedure can be used to solve for the pressure center of the normal force. At this location, the aero pitching moment goes to zero. When the pressure center is above the $\mathrm{cg}$, the moment of the normal force about the cg is equal to the moment of the gimbal force about the cg. However, the pressure center is not always above the cg, especially with the cg migrating upwards as the propellant burns. The pitching moment relative to the gimbal and cg is plotted in Fig. 9 for an early AIX trajectory. The corresponding plot of normal force and gimbal force is shown in Fig. 10. Note the gimbal force peaks at the max qalpha condition around $\mathrm{t}=56$ seconds and decreases after that as the cg moves up the vehicle and dynamic pressure drops.

The aero line load distributions used to match the wind tunnel integrated forces and moments are shown in Fig. 11. The variations were based on curves similar to Fig. 7. The sum of the lateral line loads must equal the total normal force. Also, the moment sum of lateral forces about the cg must equal the pitching moment relative to the cg. These two conditions make it possible to solve for the lateral line load unknowns w1 and w3. An example of this lateral load distribution is illustrated in Fig. 12. These curves represent normal loads for max qalpha conditions of two different trajectories. The sum of the axial force line distributions must equal the total axial aero force. Like the lateral load distribution, the axial line load distribution was influenced by the CFD line load variations. The axial aero distributions for three trajectory time points are shown in Fig. 13. Note that the maximum axial aero occurs near the max dynamic pressure at time $\mathrm{t}=52.6$ seconds. By late 2007, there was good correlation between integrated CFD aero distributions and wind tunnel results. However, the preliminary structural loads released in the spring of 2007 were based on the approximate aero line loads described above.

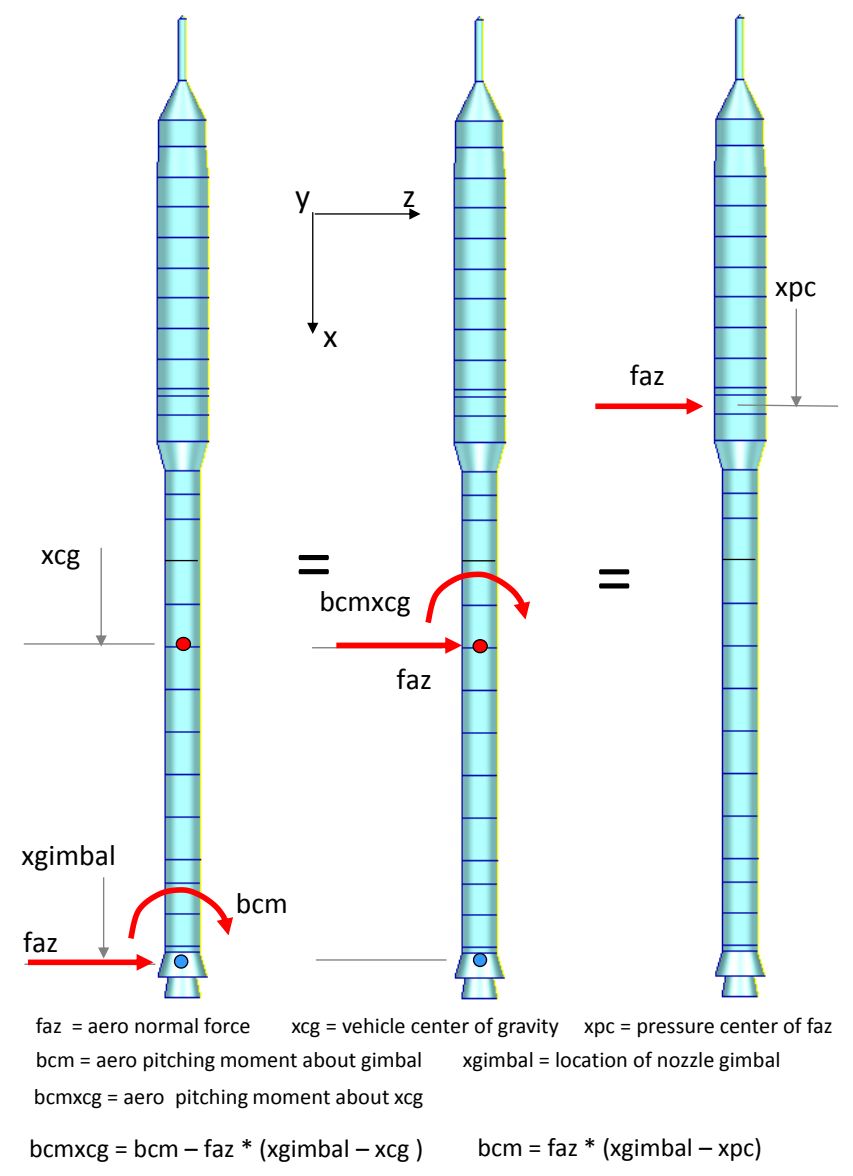

Figure 8. Equivalent Aero Force Systems. 


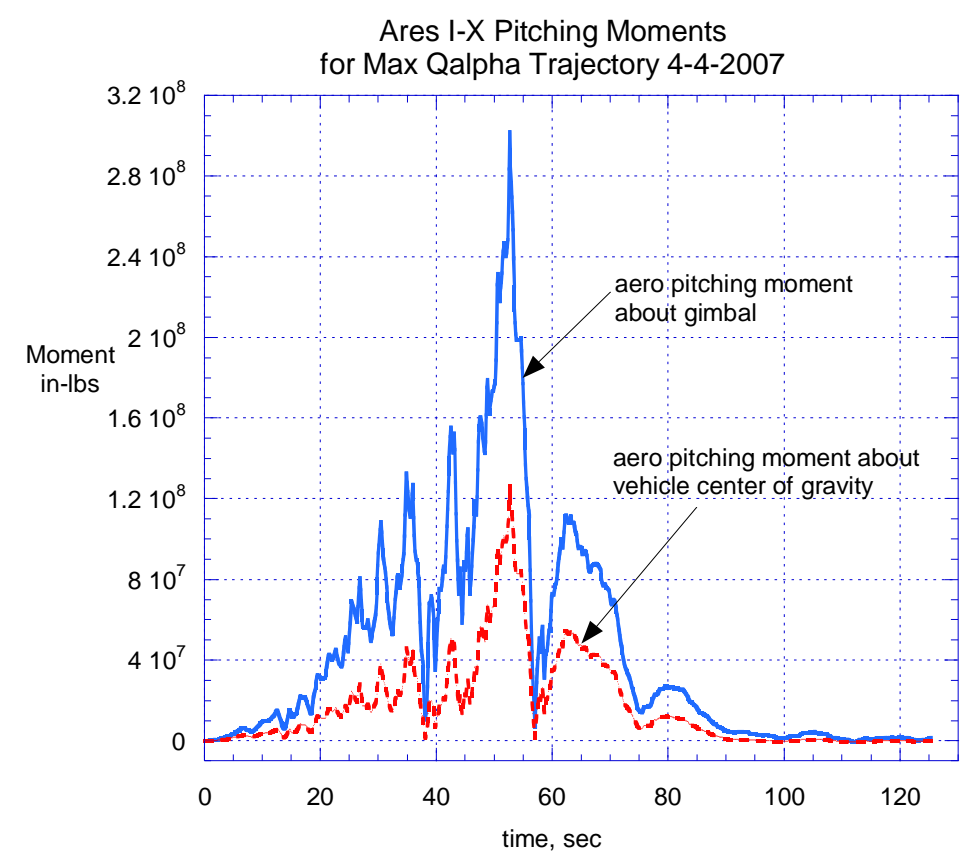

Figure 9. Aero Pitching Moment Relative to Gimbal and Vehicle CG.

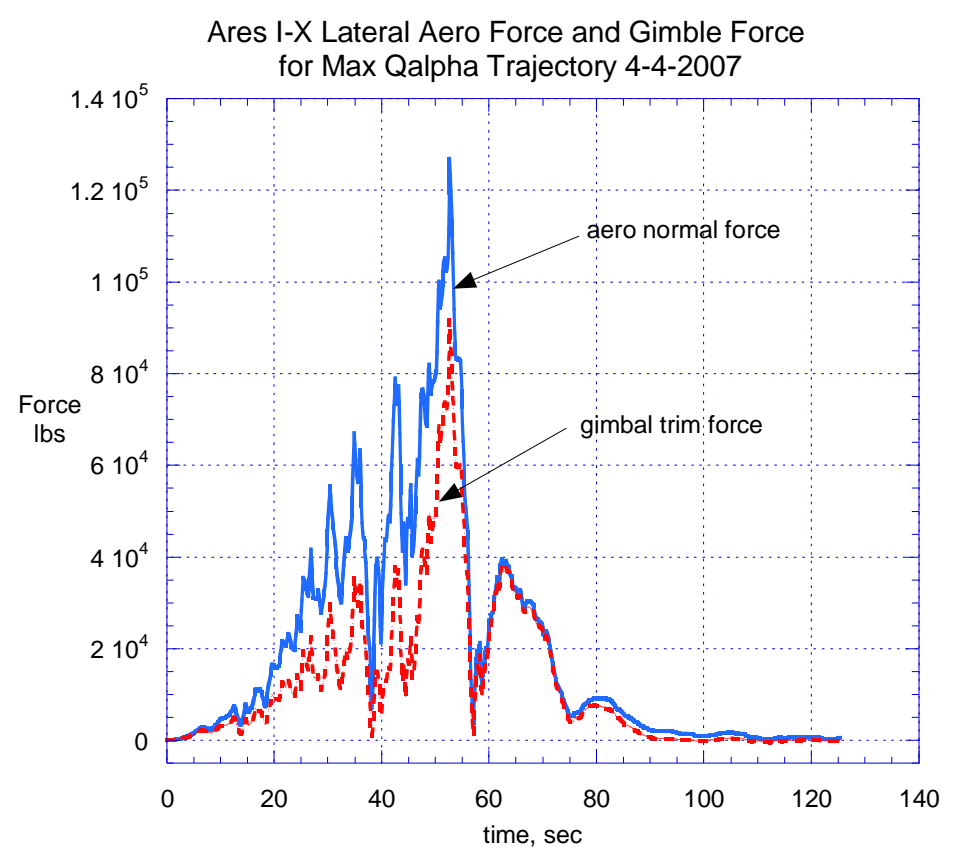

Figure 10. Aero Normal Force and Gimbal Trim Force.

American Institute of Aeronautics and Astronautics 


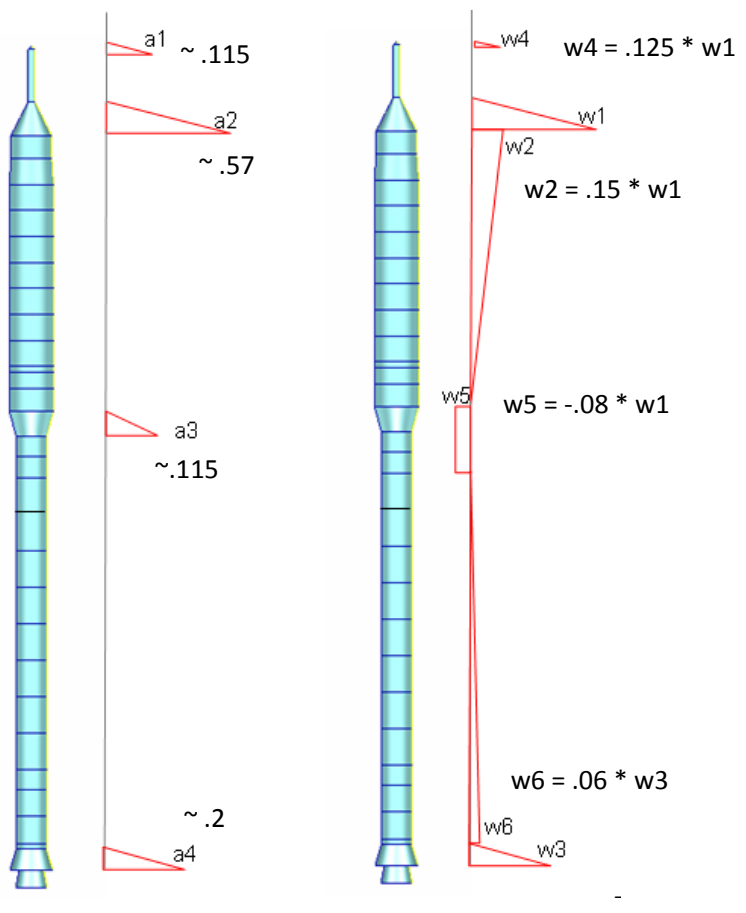

Figure 11. Assumed Aero Force Distributions.

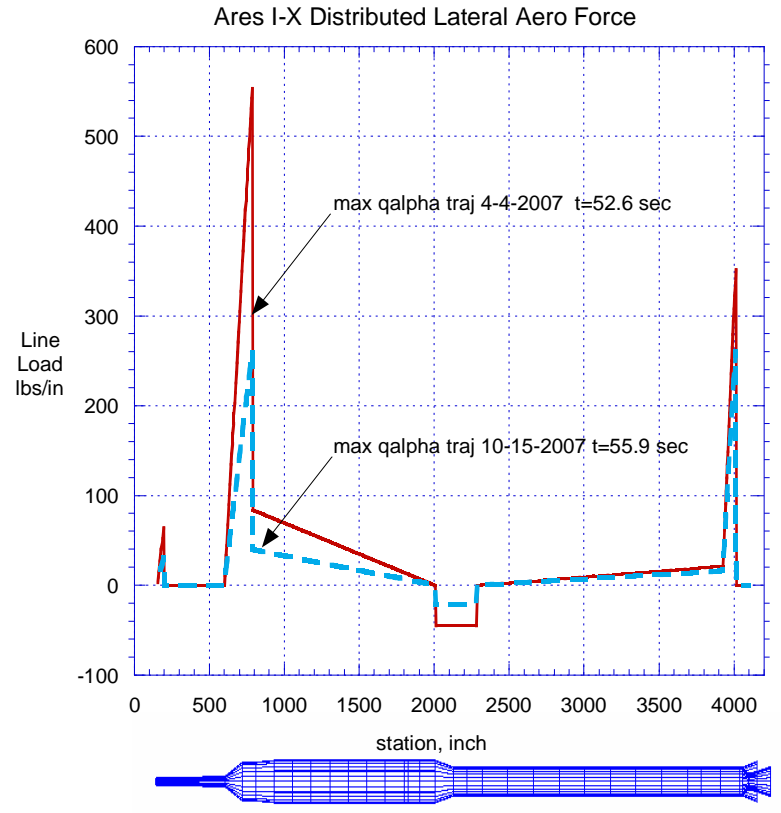

Figure 12. Lateral Aero Force Distributions.

American Institute of Aeronautics and Astronautics 


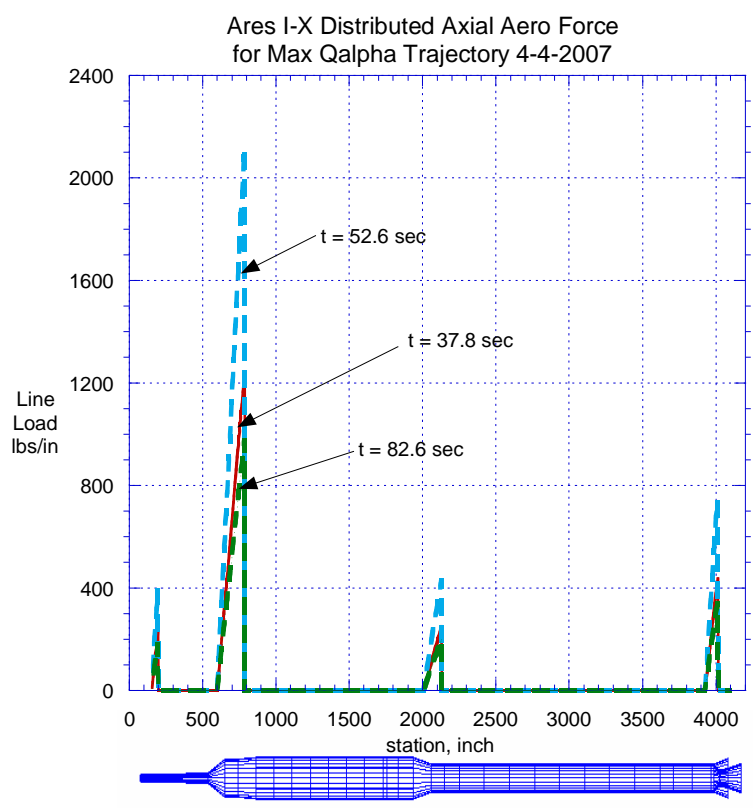

Figure 13. Aero Axial Force Distributions.

The distributed propulsion forces include motor thrust and gimbal trim forces. Thrust load for a solid motor is actually the net difference of forces acting at the fore and aft motor domes. A typical set of motor dome forces for an RSRM is detailed in Fig. 14. The fore dome is considered the point of thrust application for the solid motor. Note that forces F1 and F2 in Fig. 14 put the motor in axial tension. These forces are generated by motor pressures acting on the dome surfaces. The motor burn pressures also act on the interior cylinder walls to produce hoop tension. The solid motor casing functions as a pressure vessel in bi-axial tension. Flight loads are added to the motor pressure vessel loads. Trim loads are generated at the gimbal location by vectoring the nozzle. Tilt and rock actuators produce the nozzle vectoring and are part of the vehicle control system.

Overall vehicle equilibrium must be checked before calculating vehicle internal forces. Vehicle equilibrium under aero, propulsion, and trim forces is detailed in Fig. 15. Note that the lateral aero and trim forces balance the inertial loads produced by the sensed lateral accelerations. As noted earlier, the trim force can be estimated by enforcing zero angular accelerations for the vehicle. Aero and inertial axial forces are put in equilibrium by the thrust applied at the forward motor dome. The structure above the motor dome will be in compression while the motor casing below the dome is in tension. Estimates for vehicle internal forces will improve as the distribution of applied forces (aero, inertial, propulsion) is refined.

The distributed applied forces were mapped to the centerline of the vehicle. A line element free-body diagram is illustrated in Fig. 16. For the preliminary design loads, the aero forces were based on Mach number, roll angle, and total angle of attack. A single plane was used for the equilibrium equations instead of solving for equivalent angle of attack and sideslip angle and using two separate planes for shear and bending loads. The design loads at the bottom of the line element are a function of the end conditions from the element above and the element distributed aero and inertial forces. Line element calculations start at the CM-LAS tip where the free end initial conditions are known to be zero. The propulsion and trim forces require separate free-body diagrams because these forces are applied at discrete points. These joint loadings result in discontinuities in axial force or shear. A gimbal joint freebody diagram is detailed in Fig. 17. The transverse shear on each side of the joint will vary by the trim force. 


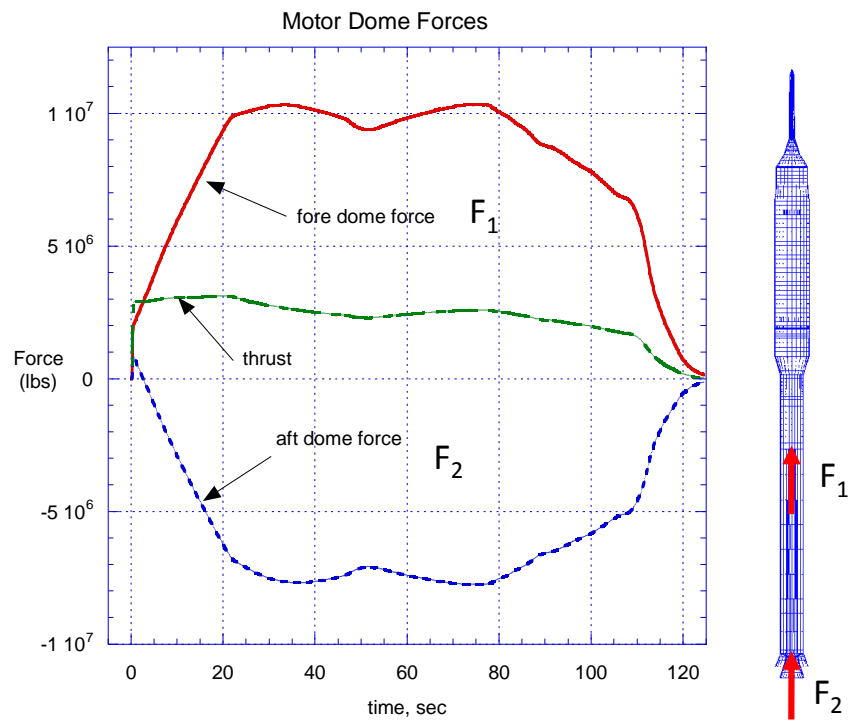

Figure 14. First Stage Fore and Aft Dome Forces

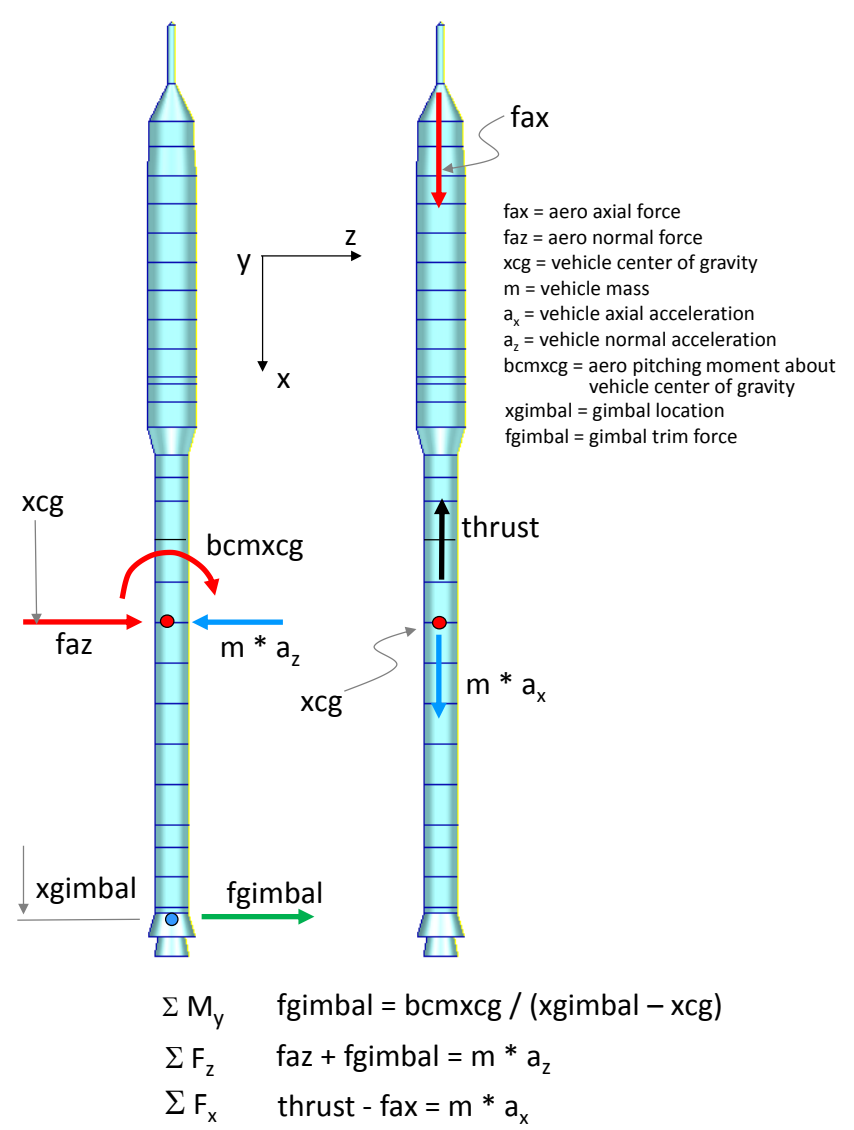

Figure 15. Overall Vehicle Equilibrium. 


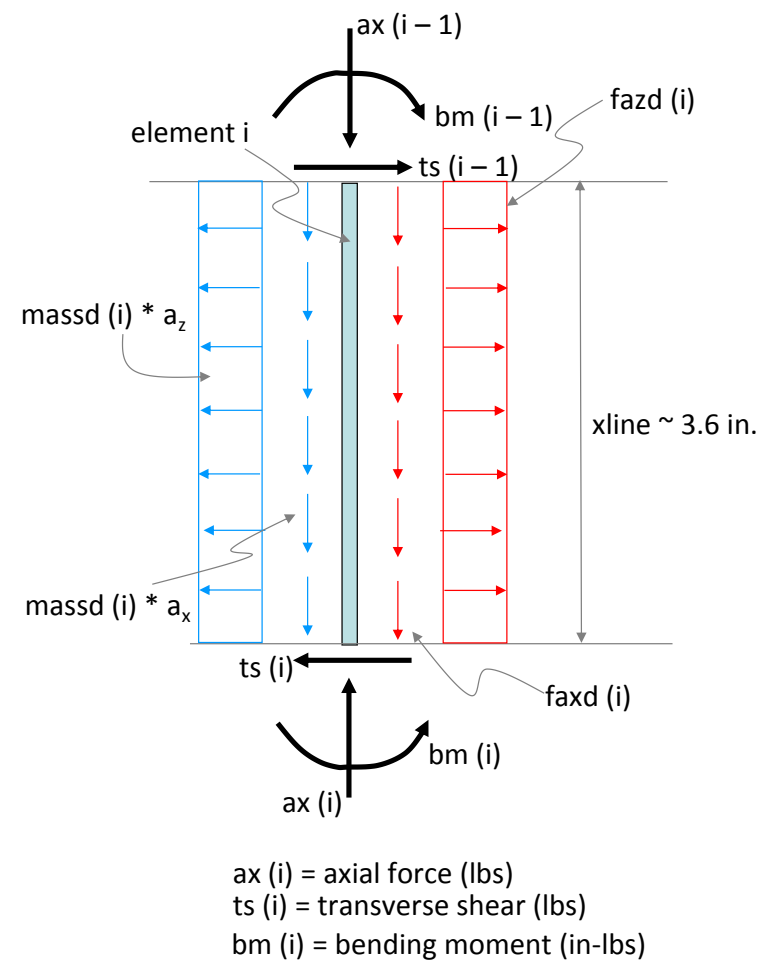

Figure 16. Free-Body Diagram of Vehicle Line Element.

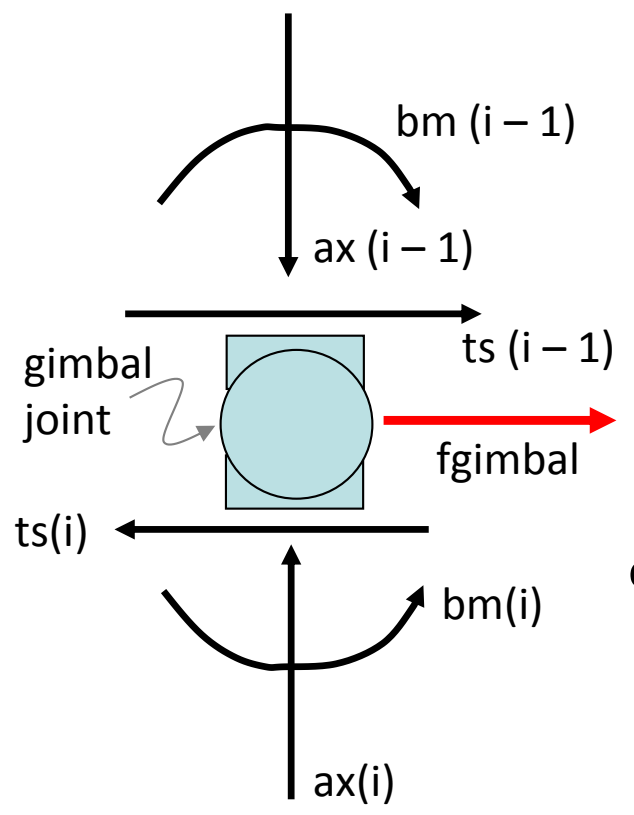

bm(i - 1) = bending moment above gimbal joint $b m(i)=$ bending moment below gimbal joint $\operatorname{ax}(i-1)=$ axial force above gimbal joint $a x(i)=$ axial force below gimbal joint ts $(i-1)=$ shear force above gimbal joint ts $(i)=$ shear force below gimbal joint fgimbal = gimbal trim force

end conditions for next segment:

$$
\begin{aligned}
& \operatorname{ax}(i)=a x(i-1) \\
& \text { ts }(i)=\text { ts }(i-1)+\text { fgimbal } \\
& b m(i)=b m(i-1)
\end{aligned}
$$

Figure 17. Free-Body Diagram of Gimbal Joint. 
A typical axial force diagram for the AIX vehicle is shown in Fig. 18. The compressive force reaches a maximum just above the forward dome joint. Axial force discontinuities occur at the forward and aft domes and are related to the dome forces shown in Fig. 14. Vehicle transverse shear forces are illustrated in Fig. 19. The maximum shear for qalpha conditions occurs just above the gimbal point where the trim force pushes the transverse shear back to zero. The bending moments associated with the shear diagrams of Fig. 19 are shown in Fig. 20. The maximum bending moment occurs at the point of zero shear near station 2350. All three internal force plots include a DLF of 1.5.

Normal stress running loads represent a time-consistent combination of axial force and bending moment. Design engineers use running loads to reduce the number of stress indexes they need to track during hardware design. The curves in Fig. 21 show maximum normal stress running loads (compression) for time points associated with maximum bending moment and maximum axial force. The normal stress running loads aft of the motor forward dome are dominated by tensile loads produced by motor pressure. The compressive running load combination from aero and inertial loads reaches a maximum of about 10,000 lbs/in just above the motor forward dome. Corresponding minimum normal stress running loads (tension) are detailed in Fig. 22. Tensile running loads were important in the design of new segment joints with pre-tensioned fasteners. The clevis-tang joints for the FS motor casing were designed for tensile running loads above AIX values.

Trajectory-based structural loads are equivalent to the structural loads from a Nastran inertia relief analysis. In both cases, solutions are based on a set of rigid-body inertial forces that keep the applied aero and propulsion forces in overall dynamic equilibrium. Loads from Nastran inertia relief analyses were used as a means of verifying the results from trajectory-based loads analyses. Bending moments from the two analysis methods are shown in Fig. 23 for a particular trajectory time point analysis. Although a different sign convention is used by the two analysis methods, there is good agreement in the comparison of bending moment magnitudes.

Results over a series of trajectories were used to develop design load envelopes by station point on the vehicle. Each trajectory had approximately 1250 time points with about 1000 output points along the vehicle. Each station output point involved six internal forces. This process generated about 7.5 million pieces of data per trajectory. Final design load envelopes were based on maximum internal force values at each vehicle station point.

The preliminary design loads from trajectory-based analyses were also compared to the loads obtained from later coupled-loads analysis (CLA). The CLA loads were based on forced dynamic response analyses using the

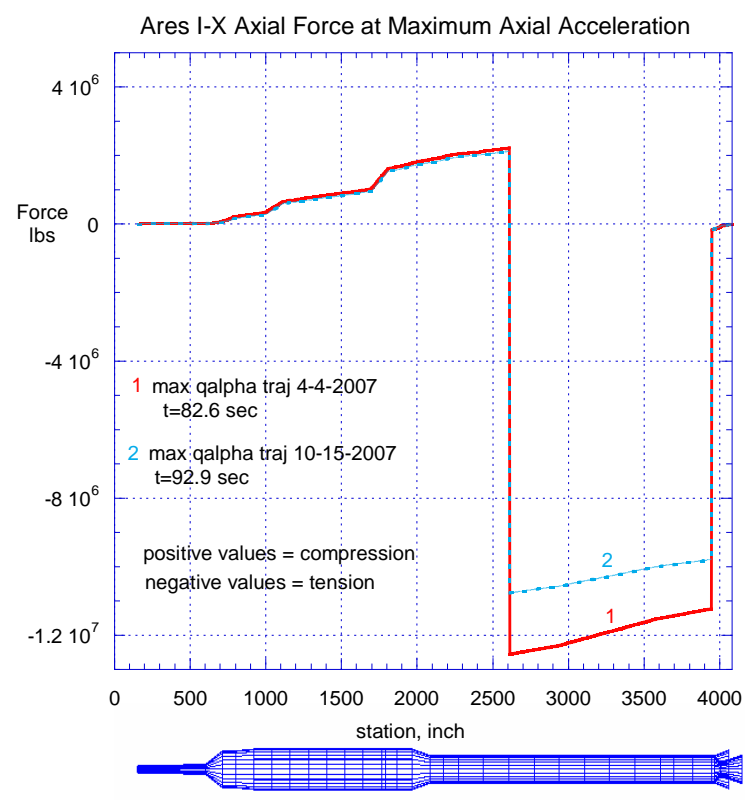

Figure 18. Vehicle Axial Force at Time of Maximum Acceleration 


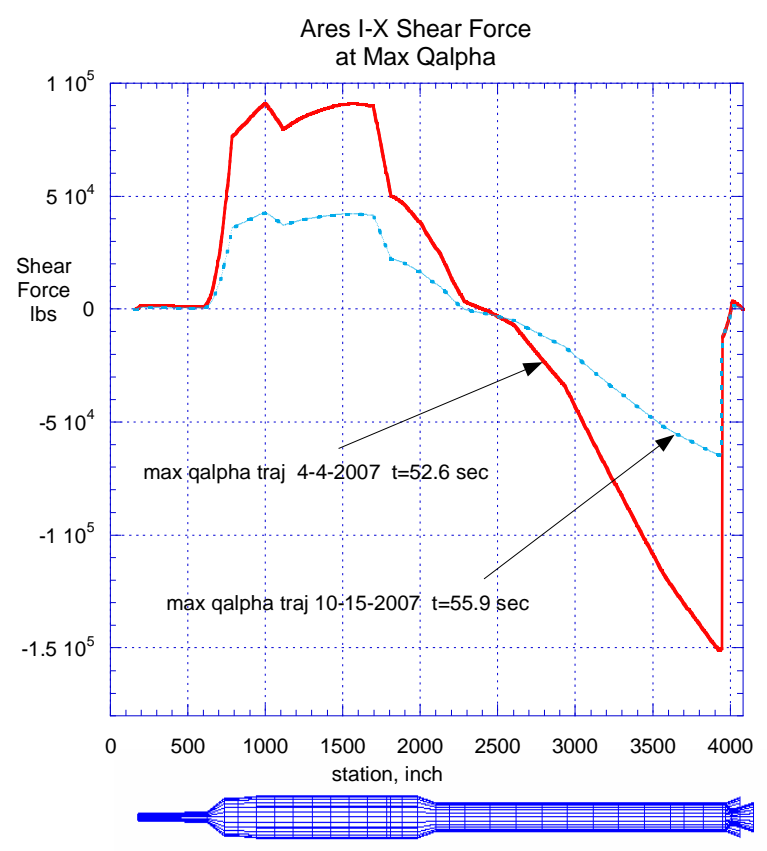

Figure 19. Shear Force at Maximum qalpha Time.

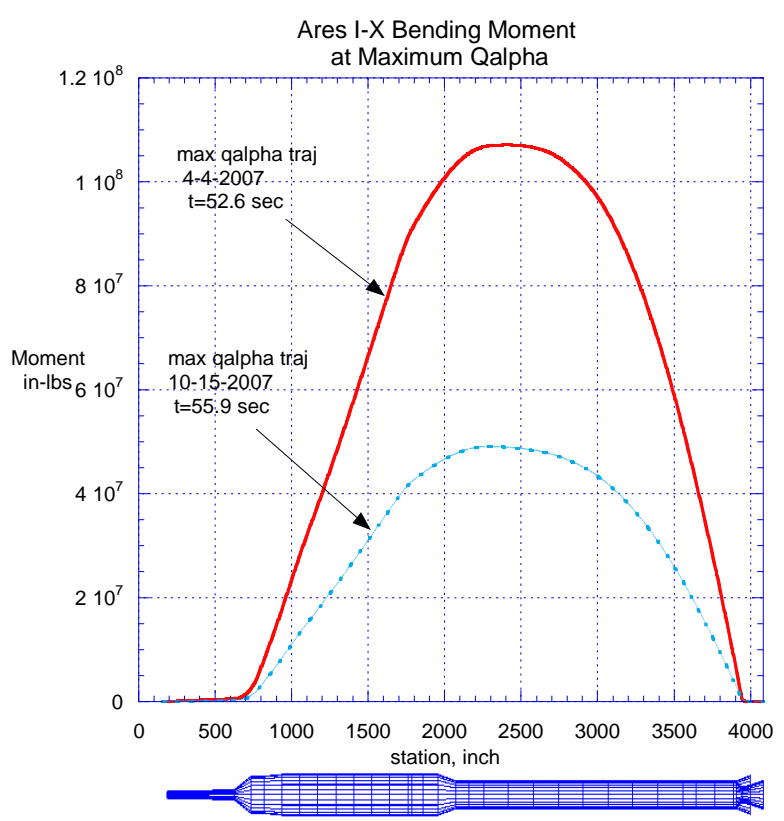

Figure 20. Bending Moment at Maximum qalpha Time.

15

American Institute of Aeronautics and Astronautics 


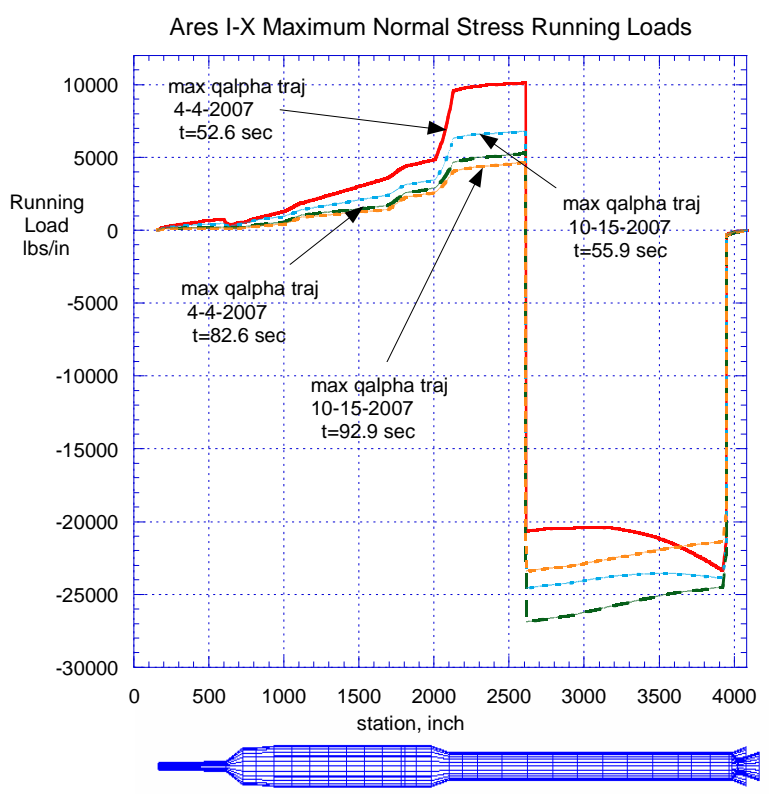

Figure 21. Maximum Normal Stress Running Loads.

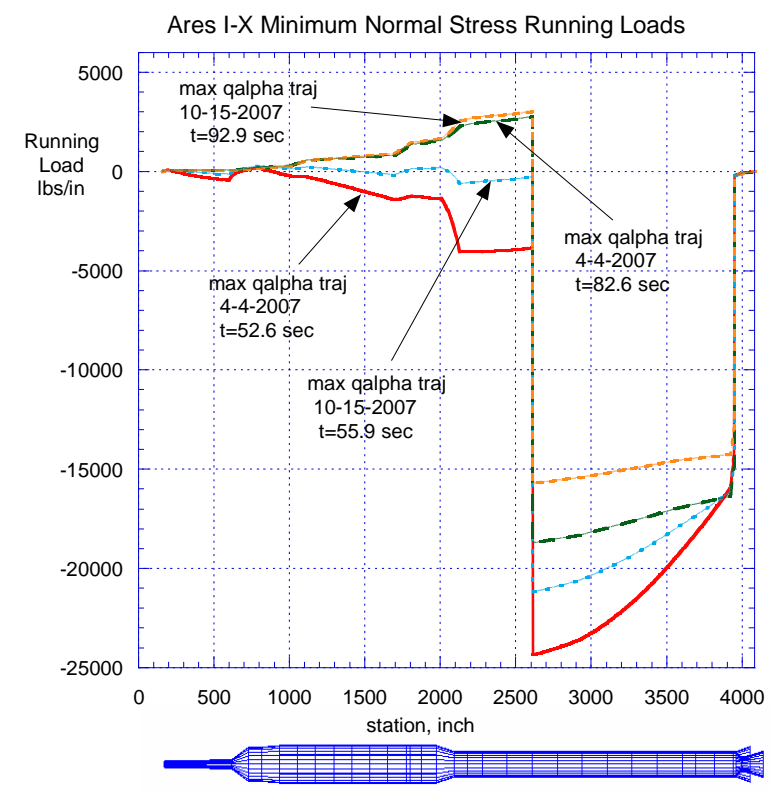

Figure 22. Minimum Normal Stress Running Loads.

American Institute of Aeronautics and Astronautics 

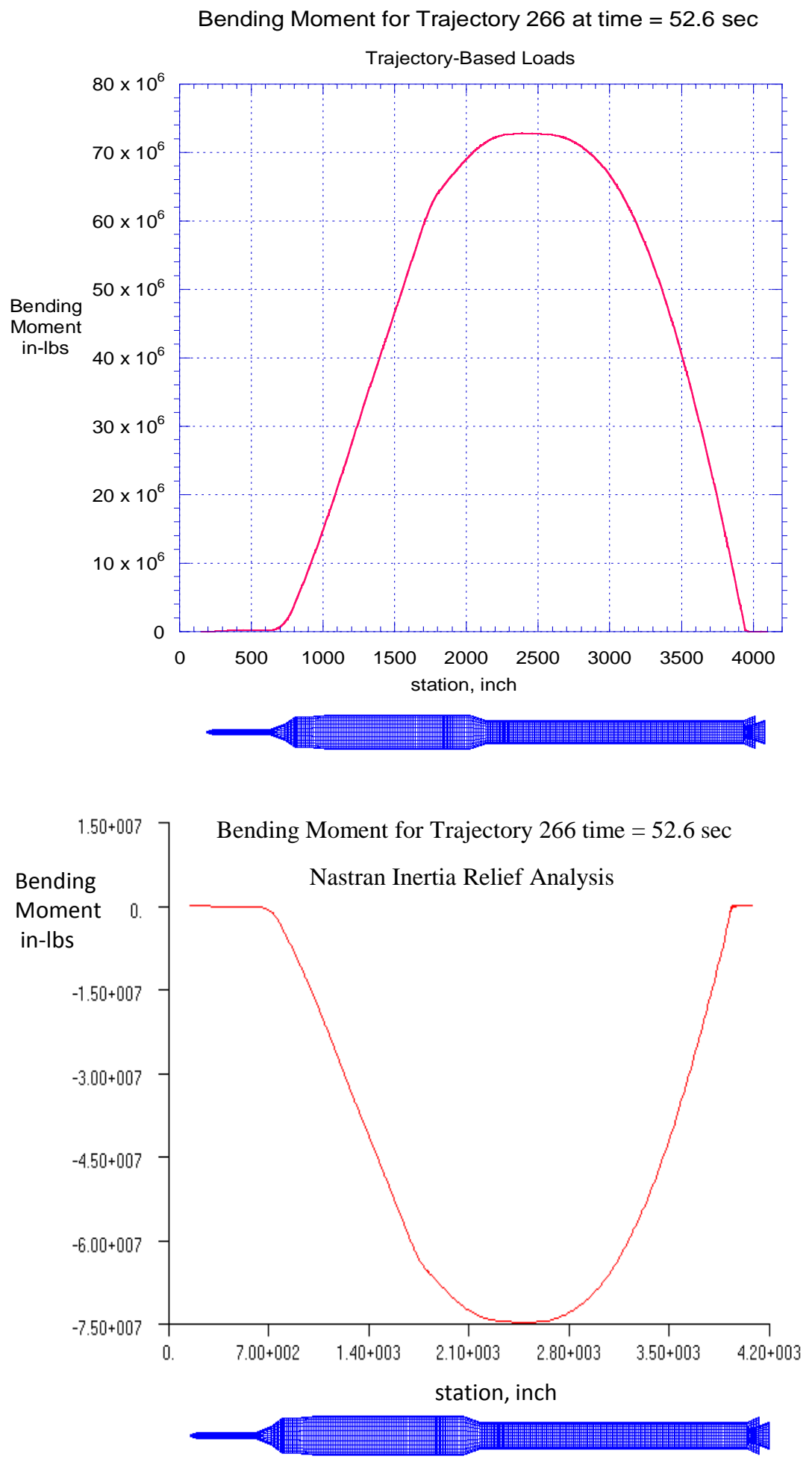

Figure 23. Comparison of Bending Moments from TrajectoryBased Loads and Nastran Inertial Relief Analysis.

17

American Institute of Aeronautics and Astronautics 
uncoupled modal method. The bending moment comparison shown in Fig. 24 is from early 2008. The trajectorybased bending moment results are labeled quasi-static in Fig. 24. There was reasonably good correlation between trajectory-based loads and formal CLA results. The CLA loads were adopted as the official design loads for AIX following the second load cycle in early 2008. However, the preliminary design load envelopes that were reported in the Summer of 2007 were obtained with trajectory-based loads methods. The section of the AIX Structures Data Book (Ref. 3) on quasi-static loads covers the development of trajectory-based loads.

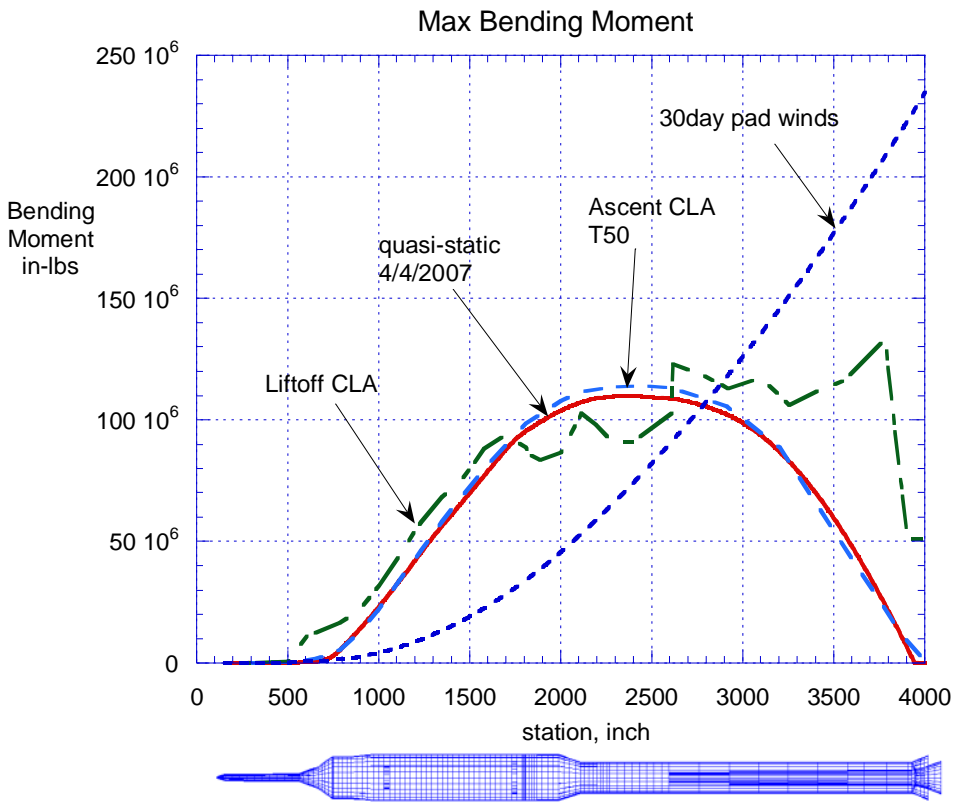

Figure 24. Comparison of Bending Moments from Coupled-Loads Analysis (Ascent CLA) and Trajectory-Based Loads (quasi-static)

\section{Range Safety Loads}

In August 2009, the AIX Trajectory Group requested a trajectory-based loads model to support their range safety malfunction turn studies. The Trajectory Group had generated a series of 8423 malfunction turn trajectories that result from possible vehicle failure scenarios following launch. The internal forces from each time point on a malfunction trajectory would be compared with the failure loads that each IPT supplied for their hardware. If the internal forces exceeded the failure loads at a specific location, then the vehicle would break into two pieces. The Trajectory Group would supply the state variables (position, velocity, and acceleration) for each piece to the USAF $45^{\text {th }}$ Space Wing at Patrick Air Force Base. This group would estimate the impact location and debris field for each of the vehicle parts.

The mass properties for the range safety trajectory-based loads were quite different than those used for the preliminary design loads. By mid 2009, each IPT had completed their hardware design and fabrication so segment masses were fairly well known. The IPTs had also developed finite element models of their hardware which matched their mass property estimates. These models were furnished to SEI, which created integrated vehicle models (IVMs) for CLA forced response analyses. The current vehicle model at the time of the range safety studies was IVM13. However, this model contained about 250,000 degrees of freedom and was far too large to be tied to a trajectory systems analysis model. The decision was made to divide each IVM13 segment into mass parts with the mass properties (including inertias) stored for each part. Each vehicle segment was divided into at least three parts. Examples of mass parts for two vehicle segments are shown in Fig. 25. A total of 119 mass parts were used in the loads model with part mass property calculations being done with Nastran. Note that FS propellant segments were treated separately from motor casing segments. Time dependent mass properties for propellant segments were based on burn weight tables from ATK. 
The sensed accelerations coming from the trajectory group were based on integrated mass properties. Therefore, integrated mass properties were calculated from the loads model distributed masses for comparison with the Trajectory Group's values. There was good correlation in comparisons of time-dependent mass properties (Figs. 26 and 27) except for roll inertia. This discrepancy was due to the large number of lumped masses at the centerline of the IVM13 model. Mass moments of inertia were not included in the lumped mass matrix for most of the IVM13 concentrated masses. The roll inertia for the loads model was adjusted to agree with the Trajectory Group's roll inertia.

Aerodynamic forces for the range safety studies were developed by the Trajectory Group as a function of Mach number, pitch angle of attack, and yaw angle of attack. The Aero Group's coefficient line distributions were integrated over each element to obtain elemental force coefficients. The elemental force coefficients were used in conjunction with the trajectory dynamic pressure to calculate forces at the 27-node range safety model (Fig. 28). Unlike the aero force development for the preliminary design loads, lateral aero forces were applied in both the xy and yz body planes of Fig. 29.

The Trajectory Group requested that multiple nodes of the range safety model be capable of handling propulsion/control force input. Some failure scenarios were being considered which could result in a breach of the FS motor casing. The jet effects at this breach would be modeled with propulsion forces. Therefore, the structural loads code was set up with full 6 degree of freedom vector input for applied propulsion forces and moments. All propulsion and trim forces for the range safety studies were generated by the Trajectory Group.

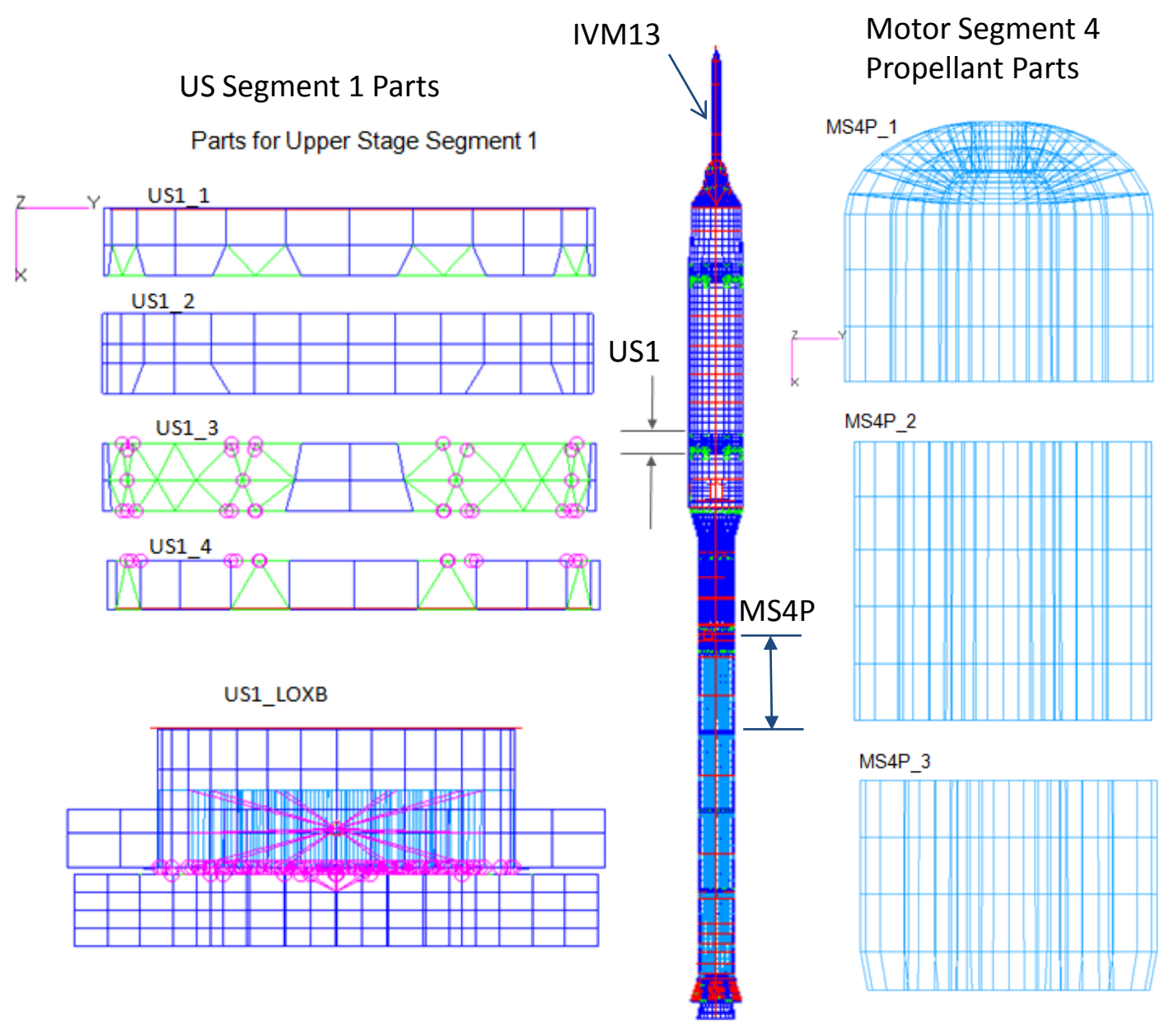

Figure 25. Segment Mass Parts from US Segment 1 and FS Motor Segment 4. 

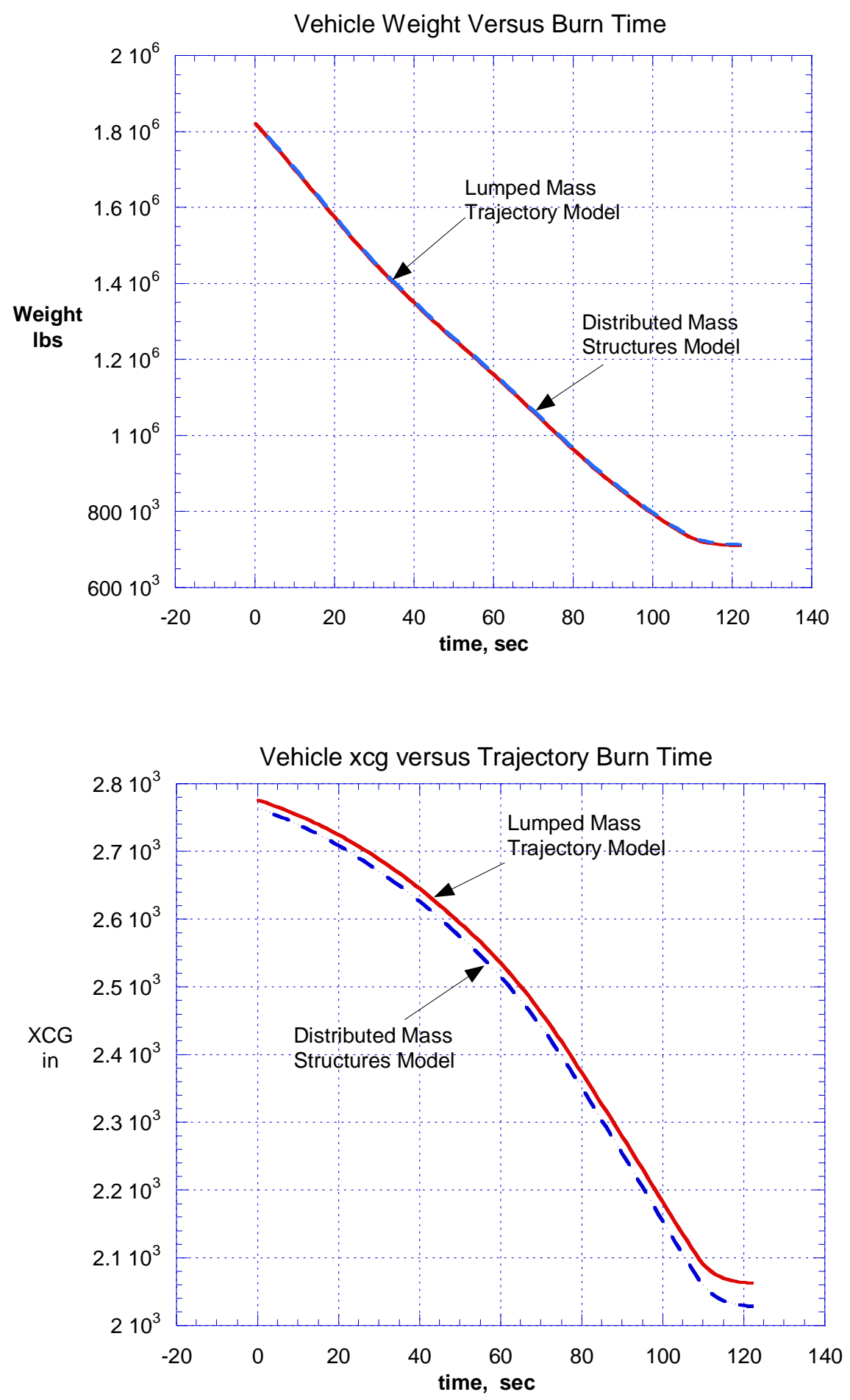

Figure 26. Comparison of Vehicle Mass and CG for Lumped Mass Trajectory Model and Distributed Mass Structures Model. 

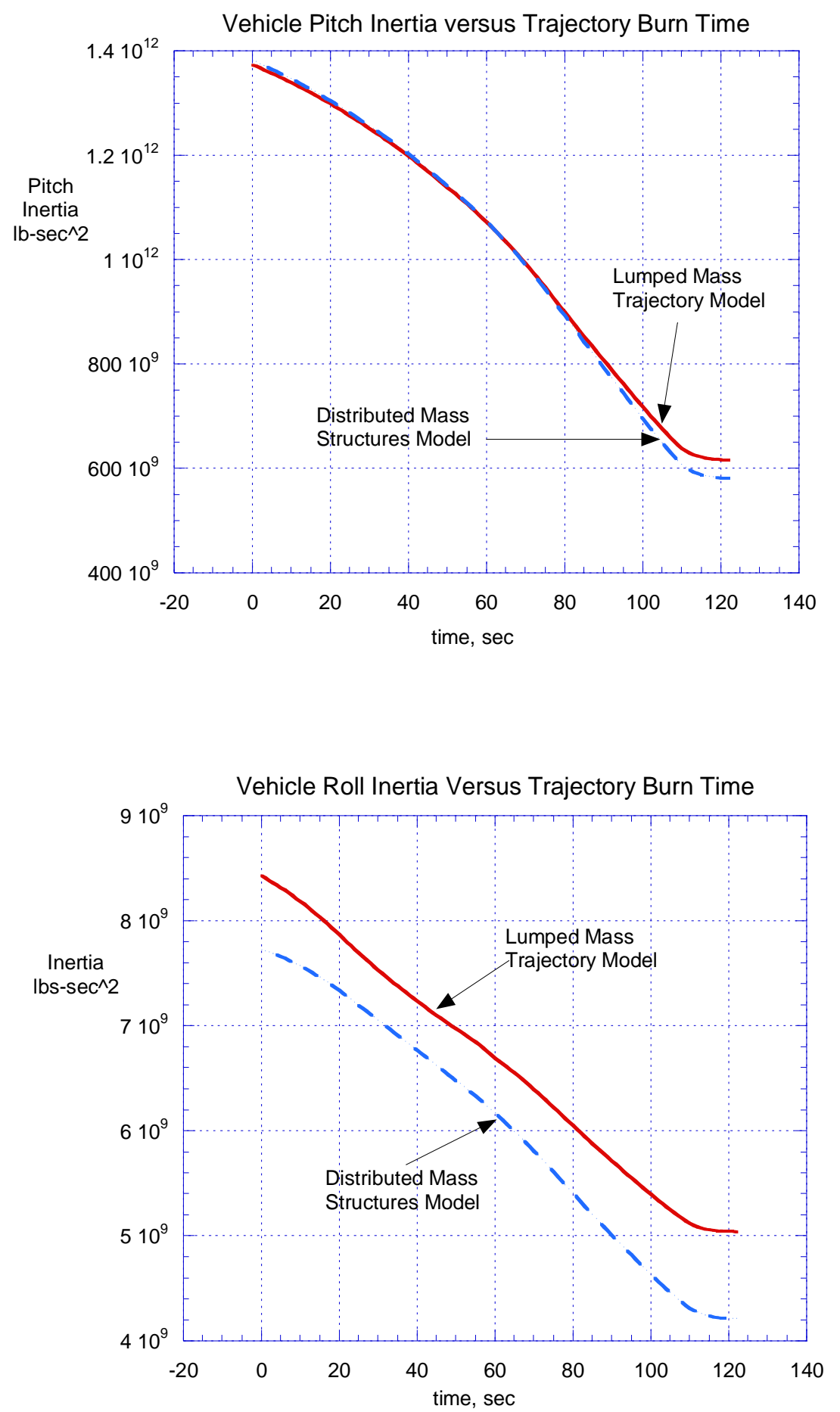

Figure 27. Comparison of Pitch Inertia and Roll Inertia from Lumped Mass Trajectory Model and Distributed Mass Structures Model. 


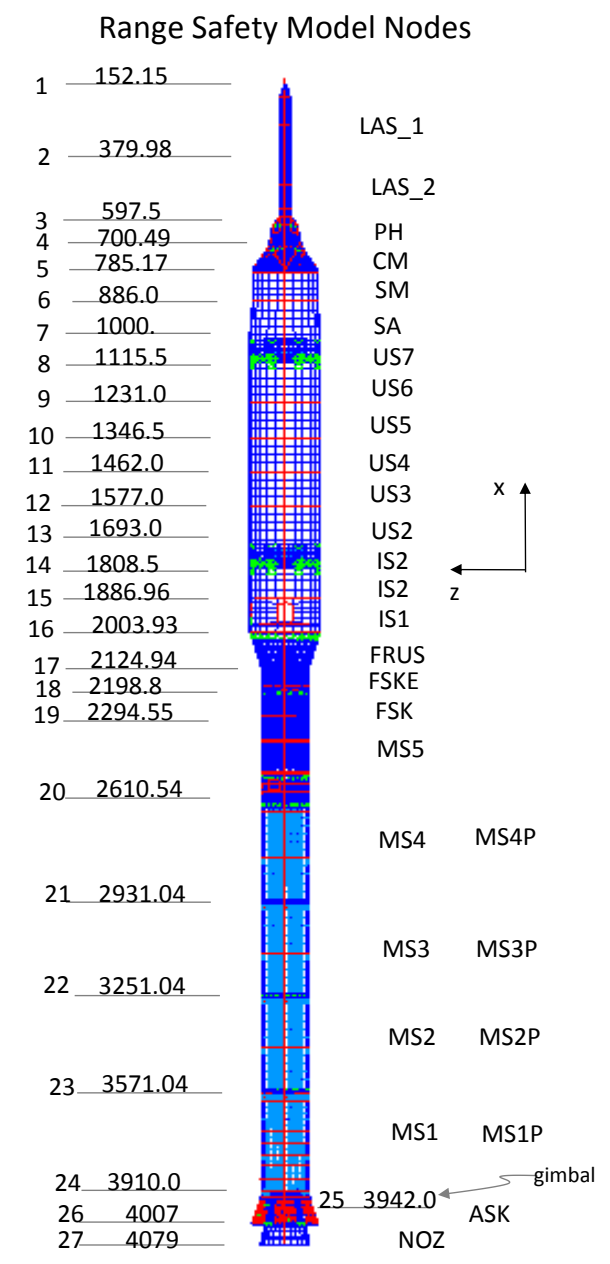

Figure 28. Node Locations for Range Safety Model.

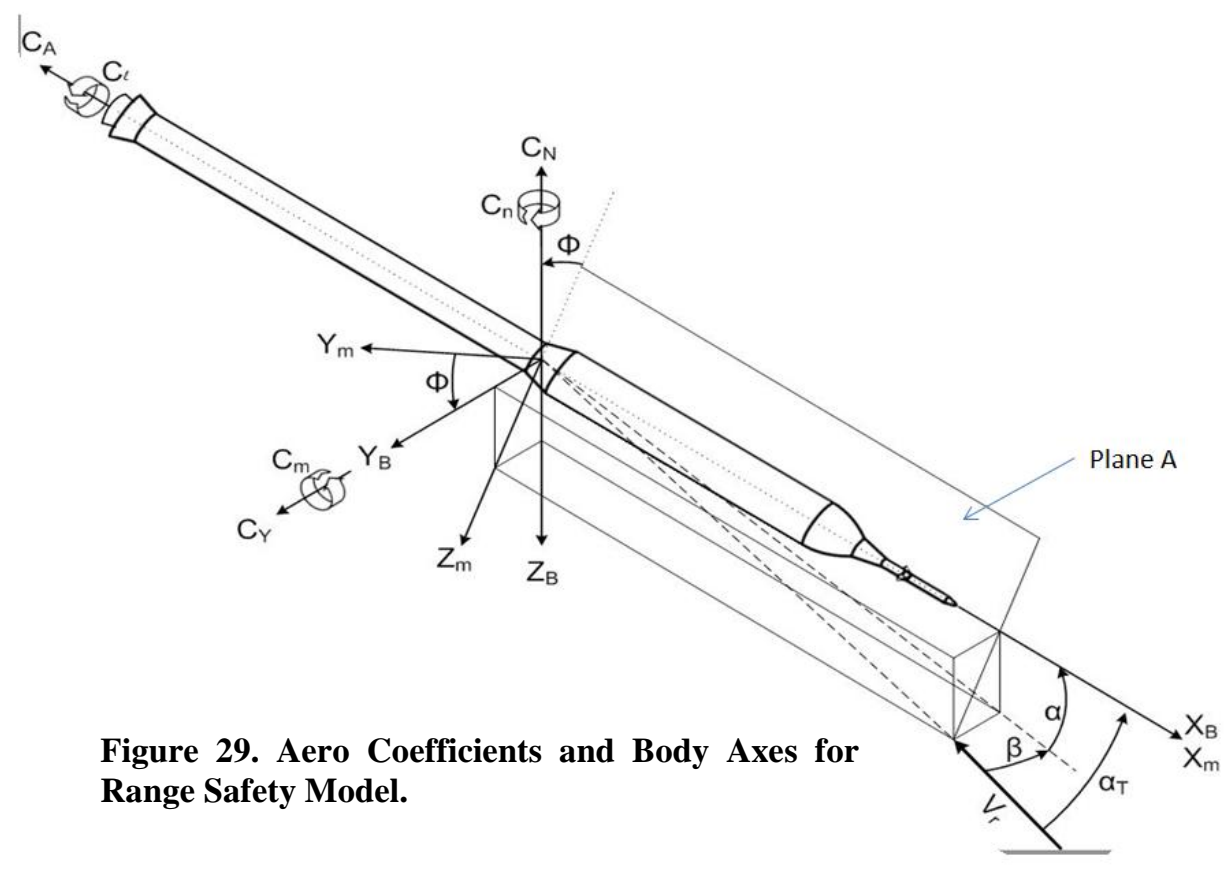

American Institute of Aeronautics and Astronautics 
Overall equilibrium of the range safety model had to be satisfied for forces and moments in three directions. The applied aero and propulsion forces were balanced by a set of inertial forces produced by vehicle translational and angular accelerations. For general cases, angular accelerations would not be affected by trim forces. Each vehicle translational and angular acceleration produced inertial forces and moments in all 119 of the mass parts. Inertial forces in the $\mathrm{xz}$ body plane are detailed in Fig. 30 for angular acceleration, angular velocity, and translational acceleration. The position of the mass part relative to the current cg determines the sign of the inertial force for vehicle angular accelerations and angular velocities. As a final check before vehicle internal force calculation, sensed accelerations were calculated using the integrated mass properties from the loads model and the nodal aero and propulsion forces supplied by the trajectory group. Comparisons were made with the acceleration vector passed from the Trajectory Group to ensure compatibility of the integrated and distributed models.

A free-body diagram for xz body plane shear and bending moment is shown in Fig. 31. Internal forces were calculated above and below each joint of the range safety model. This process was used because aero and propulsion forces could be applied at any joint. All inertia forces from mass parts and joint forces above a section cut were included in the equilibrium equations.

Test cases were run on the range safety loads model before it was tied to the malfunction turn trajectory runs. Load cases were built to check calculations for axial force, torsion, and shears and bending moments in the xy and xz planes. These load cases were evaluated for equilibrium, boundary conditions, and discontinuities at joint loads. The axial force distribution shown in Fig. 32 is for a load case with a $9.5 \times 10^{6} \mathrm{lb}$ propulsion force at the FS fore dome and $7.0 \times 10^{6} \mathrm{lb}$ opposite propulsion force at the FS aft dome. Compressive axial force in the range safety loads model is negative while tensile axial force is positive. The discontinuities in axial force at the fore dome and aft dome match the applied loads. The shear and bending moment diagrams of Fig. 33 were produced by a $100 \times 10^{3} \mathrm{lb}$ force applied at the nozzle gimbal in the y direction. This force represents a maximum trim force during a planned fly-away maneuver (FAM) at launch. Note the shear discontinuity at the gimbal and the shift of the peak bending moment toward the aft end of the vehicle. The FAM gimbal force is slightly larger than the peak trim force shown in Fig. 10 for the max qalpha trajectory of April 4, 2007. The vehicle torsion distribution for a $600 \times 10^{3}$ in-lb torque applied at the top of FS frustum is illustrated in Fig. 34. The discontinuity in torsion at station 2004 matches the applied moment which simulates a roll control torque. The variation in torsional moment is a function of the roll inertia distribution along the vehicle.

The AIX range safety loads code was written, debugged, and verified in a little over five weeks. Running loads for max normal stress, min normal stress, and max shear stress were passed to the Trajectory Group at joint stations for each time point of each malfunction turn trajectory.

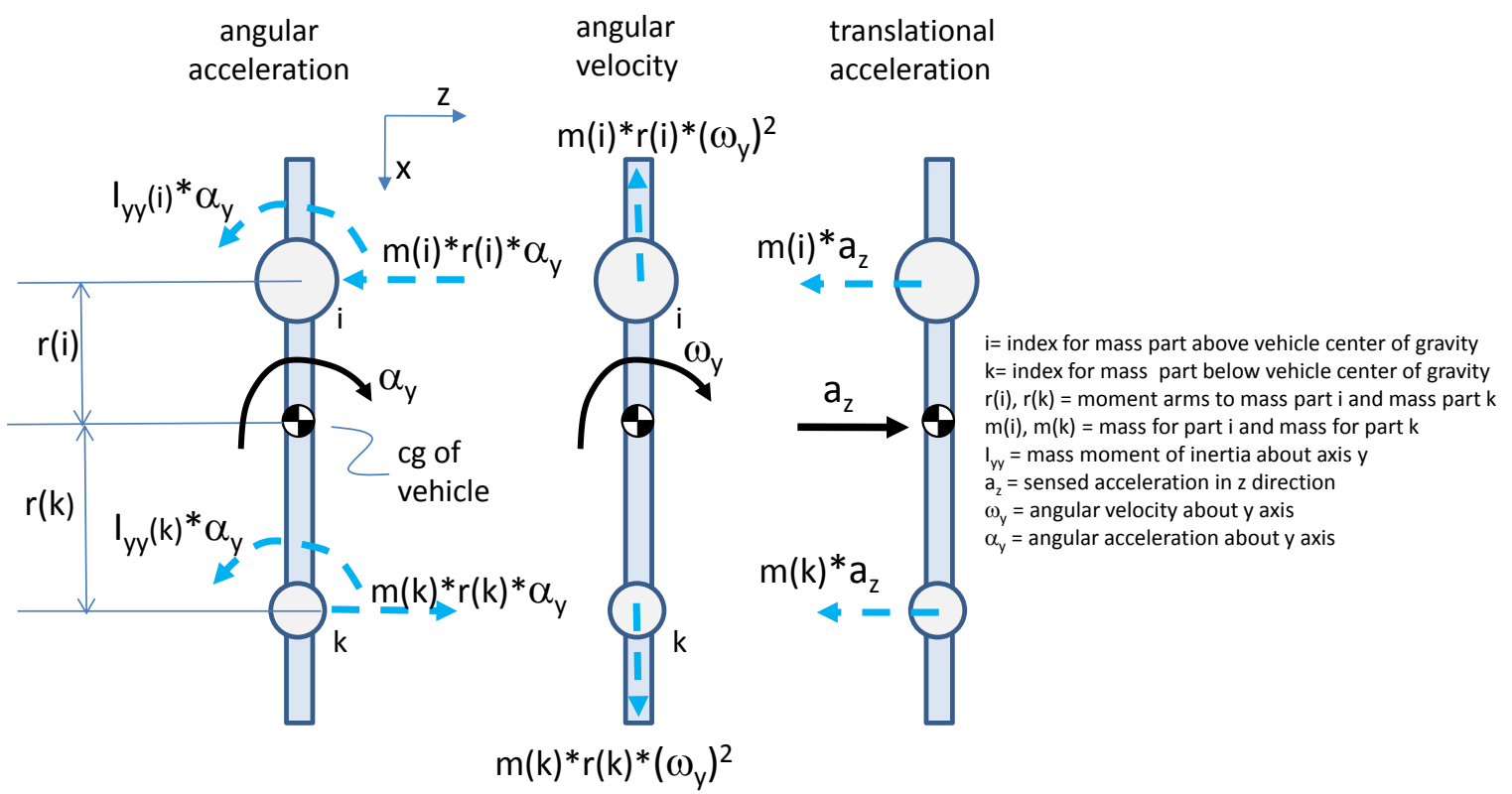

Figure 30. Inertial Load Development for Range Safety Model. 


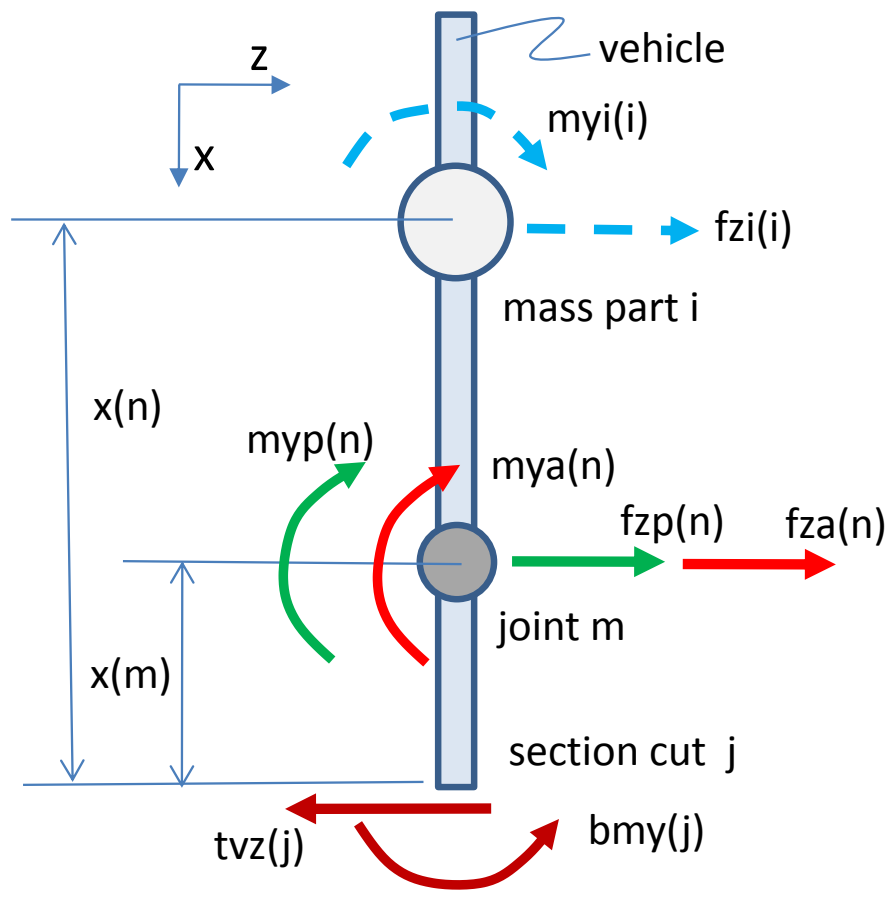

$$
\begin{aligned}
& \text { for mass part } \mathrm{i} \\
& \text { fzi }(i)=\text { inertial force } z \\
& \text { myi }(i)=\text { inertial moment } y \\
& \text { for joint } n \\
& \mathrm{fza}(\mathrm{n})=\text { aero force } \mathrm{z} \\
& \mathrm{fzp}(\mathrm{n})=\text { propulsion force } \mathrm{z} \\
& \operatorname{mya}(n)=\text { aero moment } y \\
& \operatorname{myp}(n)=\text { propulsion moment } y
\end{aligned}
$$

Figure 31. Free-Body Diagram for XZ Plane Detailing Mass Parts, Joints, and Internal Forces.

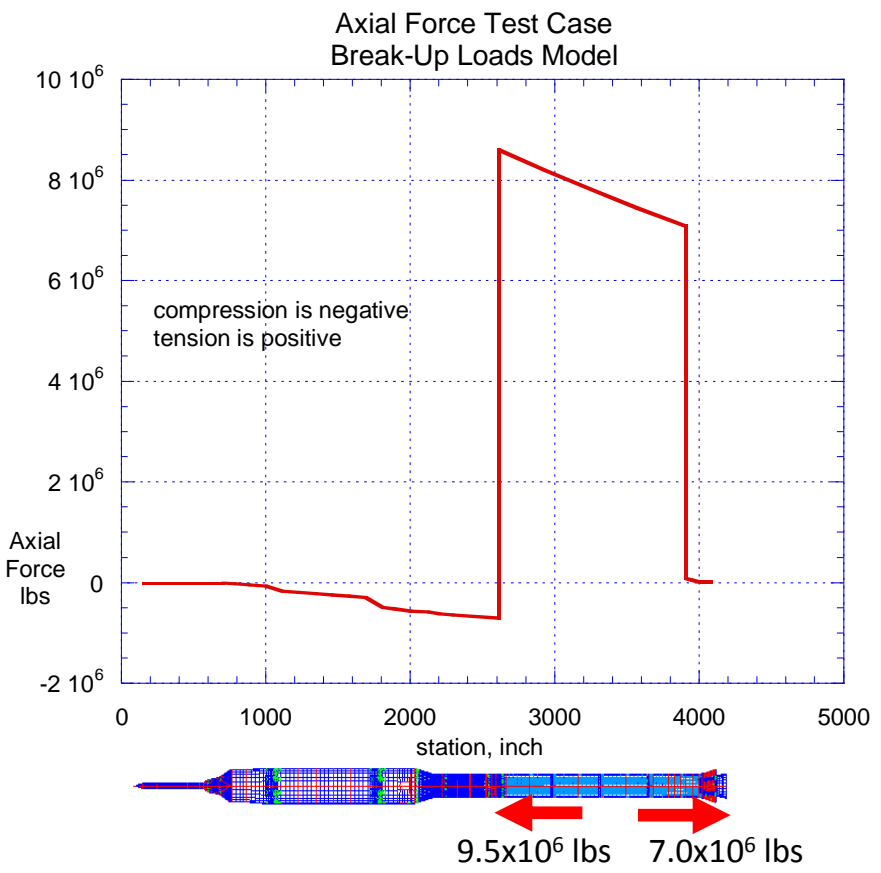

Figure 32. Motor Dome Forces for Axial Force Test Case. 


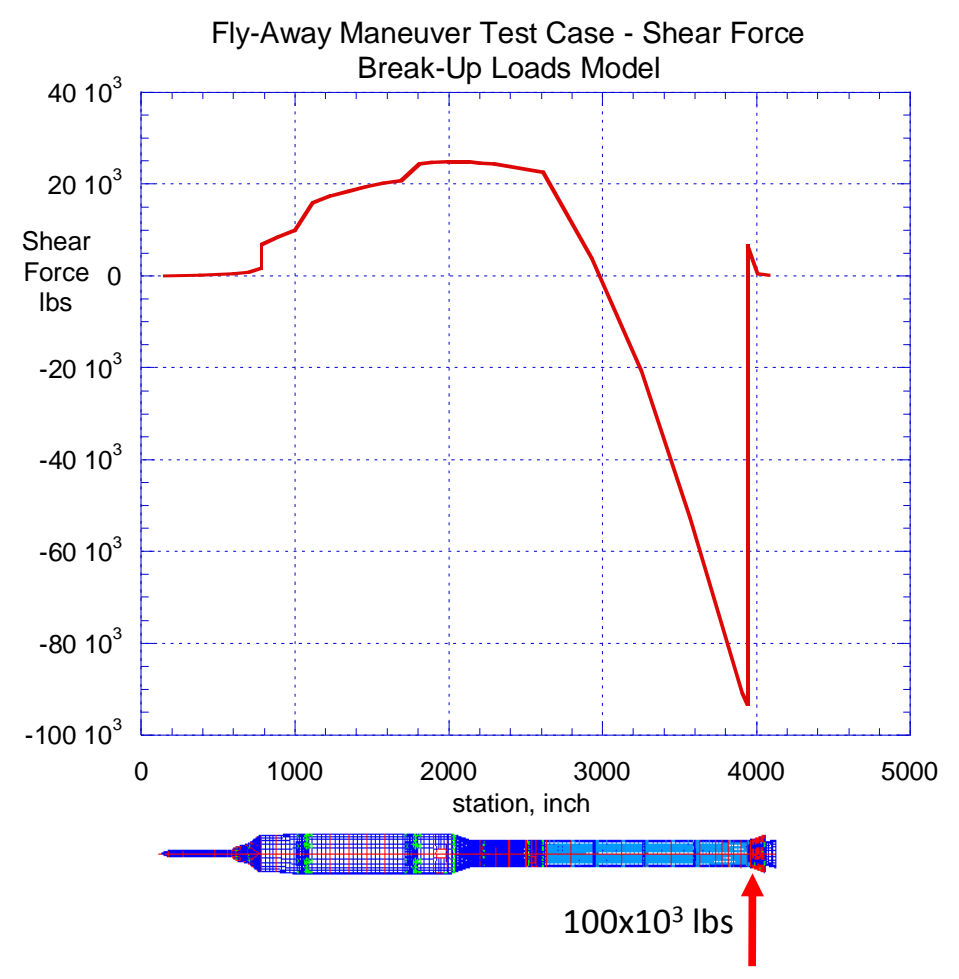

Fly-Away Maneuver Test Case - Bending Moment

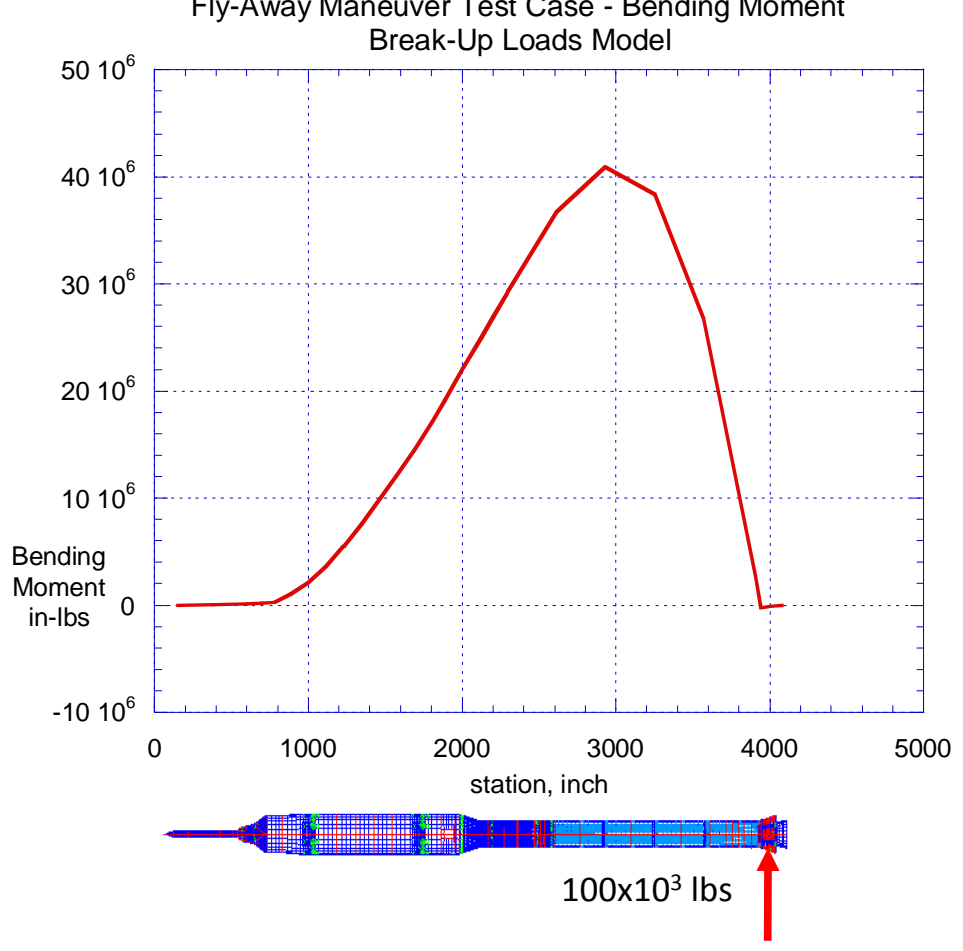

Figure 33. Fly-Away Maneuver for Shear and Bending Moment Test Case. 


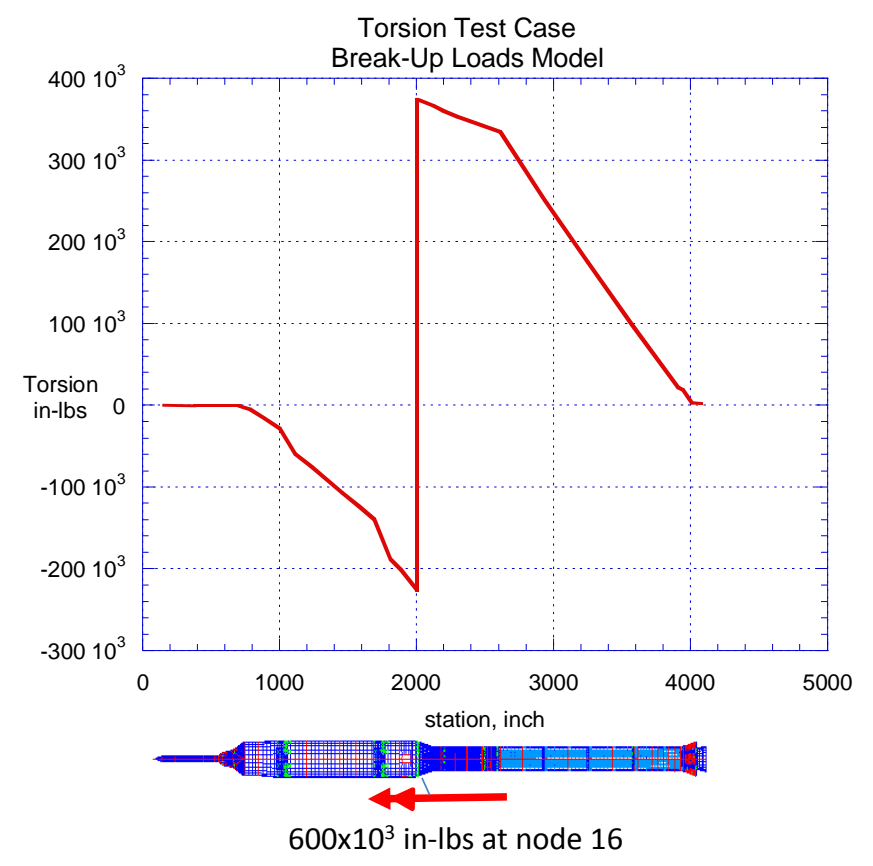

Figure 34. Roll Control Torque for Torsion Test Case.

\section{Active Control System Loads}

The AIX Guidance, Navigation, and Controls (GNC) Group used both rigid-body and flex-body dynamics models in their analyses. Lumped mass properties, integrated aero, and integrated thrust were used to predict vehicle rigid-body response (position, velocity, and acceleration). However, vehicle flex-body response was also needed by GNC to investigate the interaction of structural response frequencies with the control system (Ref. 4). The structural frequencies and mode shapes for the GNC flex-body model were based on reduced eigenvectors from the AIX Loads Group's CLA model. The CLA finite element model consisted of approximately 44000 nodes and 250000 degrees of freedom while the GNC flex-body model used 54 nodes and about 325 degrees of freedom. The reduced eigenvectors were created by focusing on vehicle response modes and average behavior at vehicle station points. The reduced eigenvectors and frequencies ensured dynamic similarity between responses from the CLA model and the GNC model. The size of the GNC flex-body model had to be kept reasonably small because it was part of an integrated systems analysis model that included mass property, aero, propulsion, controls, and trajectory models with associated uncertainties. The combinations of systems model uncertainties usually generated about 2000 Monte Carlo runs.

GNC was planning a series of low-frequency maneuvers, called planned-test-inputs (PTIs), to evaluate their control system at different AIX trajectory times. Loads recovery was requested from the Structures Group to assist GNC in designing their PTIs. The GNC flex-body model had no explicit distributed mass and stiffness models. Therefore, the decision was made to use a trajectory-based loads method to recover internal forces from the GNC systems model.

As noted earlier, the GNC model consisted of 54 nodes with the emphasis on control system sensor and effector points. Specific locations were included for the redundant rate gyro units (RRGUs), fault tolerant inertial navigation unit (FTINU), gimbal, nozzle tilt and rock actuators, roll control engines, and motor thrust application points. A series of distributed centerline nodes was included for the application of aero loads to the flex-body model. Integrated mass properties were available for the vehicle and the nozzle system. Detailed aero, propulsion, and control system forces were already defined for the GNC model. Nodal aero loads were based on the same aero 
lineloads database as the range safety loads model. The single thrust force used by GNC was replaced by fore and aft dome forces similar to the preliminary design loads model.

An explicit time-dependent mass distribution, including inertias, was defined for the GNC loads model using mass divisions along the length of the vehicle. The mass properties for each division were mapped to the 54 node model to minimize the difference between integrated GNC properties and integrated properties based on the distributed mass of the loads model.

The active control system influenced effector forces at the gimbal as well as frequency content of the inertial forces. Sensor input was checked by the control system at 50 times a second. This sampling rate produces reliable frequency content up to about $25 \mathrm{~Hz}$. The reduced eigenvectors from the CLA model included bending, axial, and torsion modes up to $25 \mathrm{~Hz}$. while the rates for effector forces at the gimbal and roll control system were generally less than $5 \mathrm{~Hz}$. Phase stabilization for the first vehicle bending mode $(1.3 \mathrm{~Hz}$. to $1.92 \mathrm{~Hz}$.) resulted in an effective first mode damping which often exceeded 10 percent ( 0.5 percent for other modes). The increased damping reduced first mode flex-body accelerations and the associated inertial forces. The loads model used total responses coming from the GNC dynamics models. The loads equations did not separate flex-body modal response from rigid-body dynamics. Any frequency content in the loads output was due to input at the 0.02 second time step dictated by the control system engineers.

Overall equilibrium was checked for the GNC loads model at each time point of each GNC trajectory. Checks with three force and three moment equations included all aero, propulsion, control, and inertial forces and moments. Any small equilibrium imbalances were removed with a correcting rigid-body acceleration vector. The inertial forces from this correcting vector used the same process developed for the range safety inertial forces. Without the correcting vector, there could be small discrepancies in closing force and moment diagrams to meet vehicle boundary conditions.

Section load calculations for the GNC model centered on nodal equilibrium. Aero, inertial, propulsion, and control forces were all applied at the joints of the GNC loads model. Internal forces were based on free-body diagrams and calculated on each side of the joints to catch discontinuities in force and moments due to applied joint forces.

Load calculations for the GNC model were not completed prior to the launch of the AIX vehicle. However, preflight simulations were used during verification runs of the GNC loads process. All calculations for the GNC loads model were done in Matlab to facilitate data transfer between the GNC Group and the Structures Group.

Bending moments based on the GNC loads model are shown in Fig. 35 for an entire pre-flight trajectory. This plot format is very useful for showing the relative moment magnitudes of flight events such as the FAM, gravity turn, and PTIs. Maximum bending moments by time are illustrated in Fig. 36 for an entire set of 2000 Monte Carlo trajectories. The 2000 dots at each time point represent maximum bending moments from the Monte Carlo runs. A plot of bending moments from the simulation of the test flight best-estimated trajectory (BET) is also shown on this figure. Note that moments for the pre-flight simulations exceed BET bending moments everywhere except at the PTI around ninety seconds. 


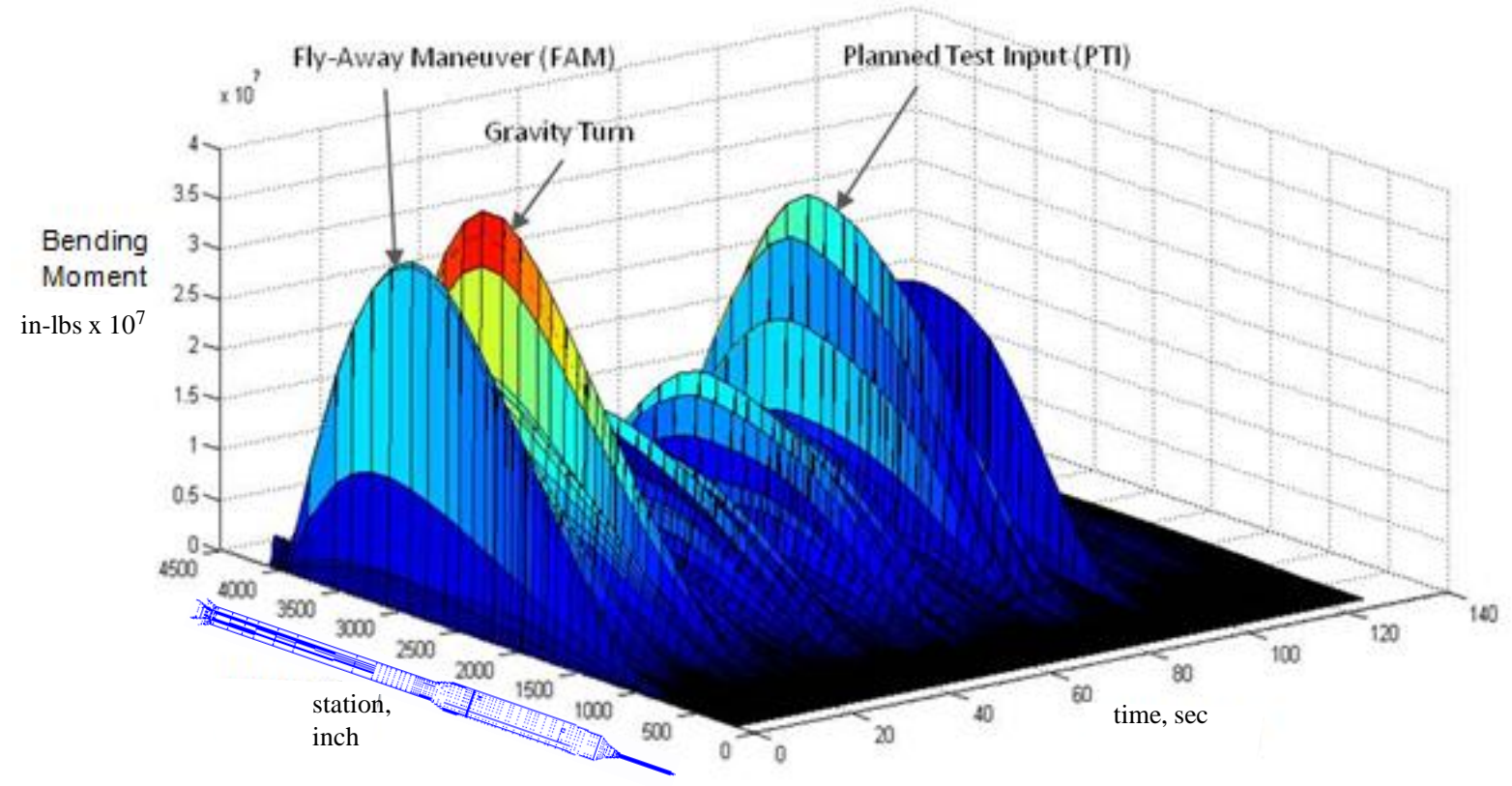

Figure 35. Vehicle Bending Moment for Each Time Point of a Trajectory.

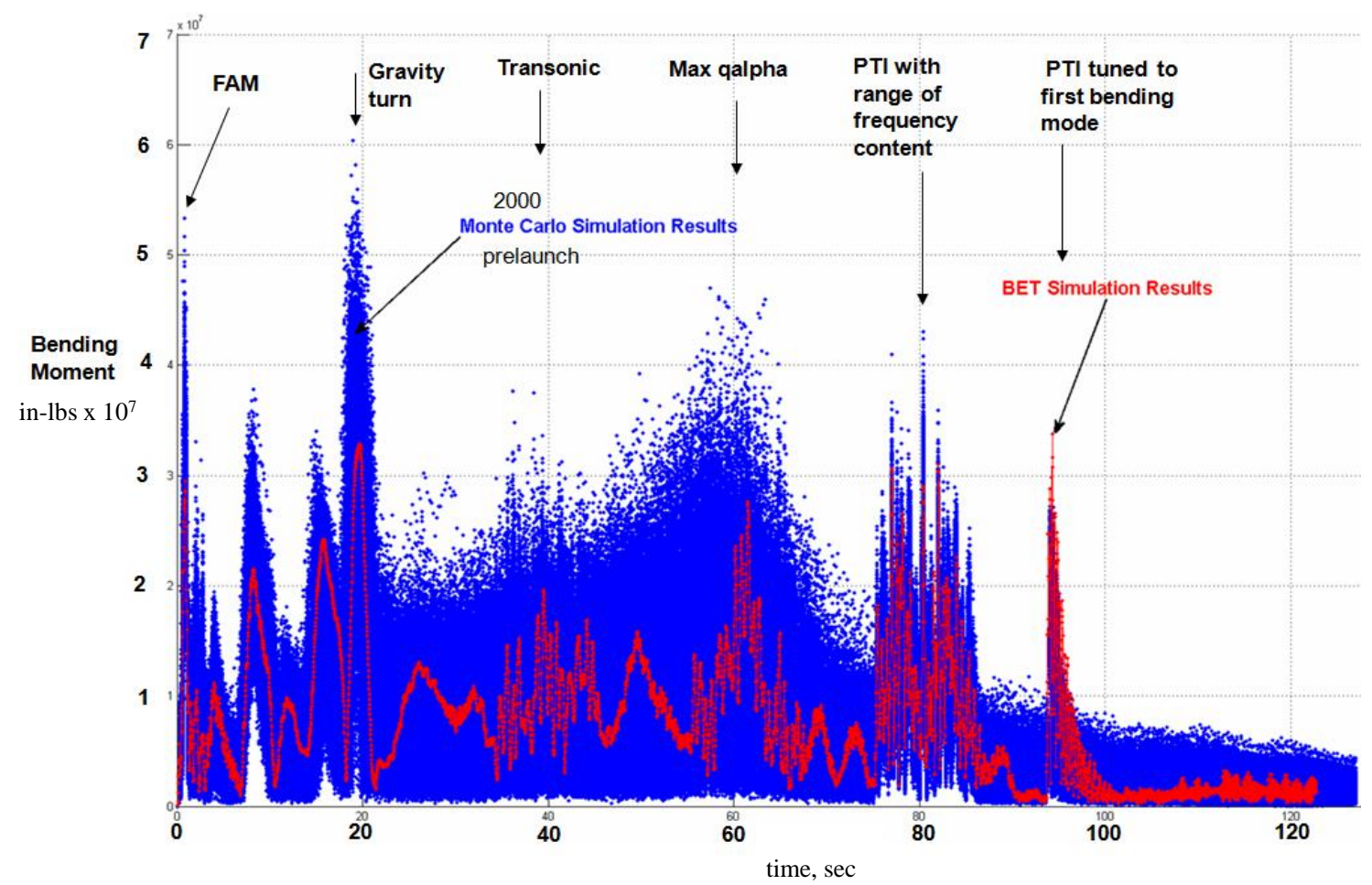

Figure 36. Comparison of Maximum Bending Moments from 2000 Prelaunch Monte Carlo Trajectories and a Simulation of the Test Flight Best Estimated Trajectory. 


\section{Conclusion}

Trajectory-based loads methods were used in the AIX Program for estimates of preliminary design loads, range safety break-up loads, and active control system loads. In each application, structural models were developed which fit within existing trajectory systems analysis models.

The structural loads model developed for AIX preliminary design loads was attached to a set of 2000 Monte Carlo trajectories. These trajectories were the result of uncertainty modeling in aero, mass properties, propulsion, and atmosphere models. The structural model could be updated quickly to accommodate a rapidly-changing design environment. There was reasonably good agreement between the trajectory-based loads and the loads results from detailed coupled-loads analyses later in the program.

The structural loads model for AIX range-safety studies was integrated with the 8423 malfunction turn trajectories. The distributed mass properties were based on a detailed model from SEI and the IPTs. Translational accelerations, angular accelerations, and angular velocity were used in the development of inertial forces for the break-up loads calculations. The structural loads for the range safety studies were tied into the 27 -node trajectory systems model in about five weeks.

The structural loads model for GNC was developed for 2000 Monte Carlo trajectories with aero, mass property, propulsion, and control system uncertainties. The loads model was adapted to fit the 54-node GNC systems model. The applied inertial forces and the control system forces were influenced by the flex-body GNC dynamics model. The GNC loads model was not completed prior to AIX launch. Pre-flight Monte Carlo runs and BET simulations were used to evaluate the GNC loads model.

In all three applications, compact trajectory-based loads models were tied to systems analysis models which executed thousands of times for Monte Carlo dispersions or postulated failure scenarios. The trajectory-based loads methods ensured consistency between lumped parameter trajectory models and the distributed forces on the structural model. As part of the integrated systems analysis, the structural calculations also included the effects of the integrated uncertainty model. Without trajectory-based loads, the structures discipline would not have been a part of these trajectory systems analysis models.

\section{References}

${ }^{1}$ Bruhn, E.F., Analysis and Design of Flight Vehicle Structures, $2^{\text {nd }}$ ed., Jacobs Publishing, Inc., Indiana, 1973, Chaps. A13A16.

${ }^{2}$ Biggs, J.M., Introduction to Structural Dynamics, $1^{\text {st }}$ ed., McGraw-Hill, New York, 1964, Ch. 2, p. 46.

${ }^{3}$ Ares I-X Systems Engineering and Integration Office., "Ares I-X Flight Test Vehicle Structures Data Book, Rev. 6.1, AI1SYS-SDB, NASA Langley Research Center, Virginia, September, 2009.

${ }^{4}$ National Aeronautics and Space Administration, "Effects of Structural Flexibility on Launch Vehicle Control Systems," NASA SP-8036, February, 1970. 